\title{
Defects and D-brane monodromies
}

\author{
Ilka Brunner ${ }^{1}$, Hans Jockers, ${ }^{2}$ and Daniel Roggenkamp ${ }^{3}$ \\ ${ }^{1}$ Institut für Theoretische Physik, ETH Zürich, \\ CH-8093 Zürich, Switzerland \\ brunner@itp.phys .ethz.ch \\ ${ }^{2}$ Stanford Institute for Theoretical Physics, \\ Stanford, CA 94305-4060, USA \\ jockers@stanford.edu \\ ${ }^{3}$ Department of Physics and Astronomy, Rutgers University, \\ Piscataway, NJ 08855-0849, USA \\ roggenka@physics.rutgers.edu
}

\begin{abstract}
In this paper D-brane monodromies are studied from a world-sheet point of view. More precisely, defect lines are used to describe the parallel transport of D-branes along deformations of the underlying bulk conformal field theories. This method is used to derive B-brane monodromies in Kähler moduli spaces of non-linear sigma models on projective hypersurfaces. The corresponding defects are constructed at Landau-Ginzburg points in these moduli spaces where matrix factorization techniques can be used. Transporting them to the large volume phase by means of gauged linear sigma model we find that their action on B-branes at large volume can be described by certain Fourier-Mukai transformations which are known from target space geometric considerations to represent the corresponding monodromies.
\end{abstract}

e-print archive: http://lanl.arXiv.org/abs/0806.4734 


\section{Contents}

$\begin{array}{lll}1 & \text { Introduction } & 1079\end{array}$

1.1 D-brane monodromies in Kähler moduli space 1082

1.2 Bulk perturbations and defects 1084

2 B-type defects in Landau-Ginzburg models and matrix factorizations $\quad 1089$

3 Gauged linear sigma models and matrix factorizations 1094

3.1 B-type D-branes in GLSMs 1094

3.2 Reduction to Landau-Ginzburg models 1095

3.3 Reduction to large volume 1096

3.4 Example: tensor product branes 1096

$\begin{array}{llr}3.5 & \text { Defects } & 1103\end{array}$

4 Landau-Ginzburg monodromy 1105

4.1 Landau-Ginzburg phase 1105

4.2 Lift to the GLSM 1109

4.3 Transport to the large volume phase 1112

4.4 Conifold and large-volume monodromies in the GLSM moduli space $\quad 1116$

5 Conifold monodromy $\quad 1119$

5.1 Conifold-like defects in general CFTs 1121

5.2 Conifold defects in the Landau-Ginzburg phase 1123

5.3 Transport to the large volume phase 1125

6 Discussion 1126 
A Dual matrix factorizations and dual complexes

\section{Introduction}

As is well known, D-branes in a given closed string background form a category, with morphisms given by open strings stretching from one D-brane to another. An important tool in the study of such D-brane categories are functors between them. Examples of such functors are T-duality, mirror symmetry and the monodromies generated by transporting D-branes around singular points in moduli spaces of Calabi-Yau compactifications.

From a world-sheet point of view, D-branes are described by boundary conditions in the conformal field theory associated to the closed string vacuum under consideration, and natural operations on them arise from defects. Defect lines are one-dimensional junctions separating possibly different conformal field theories (CFTs) ${ }^{1}$ on the same world-sheet (see, e.g., [2-10] for more details on defects in conformal field theories). Pushing such a defect line onto world-sheet boundaries, a new boundary condition is created out of the one originally imposed there. For general defects this fusion process is singular (see, e.g., [1] for an example in the free boson CFT), but if defects and boundary conditions preserve the same $N=2$ superconformal symmetry, it can be regularized and effectively described in the topologically twisted theory $[11,12]$. In this way, supersymmetry preserving defects give rise to functors between D-brane categories.

The functors we will consider in this article are monodromy transformations obtained by transporting B-type D-branes around singularities in Kähler moduli space of $N=2$ supersymmetric non-linear $\sigma$-models. This in particular includes the monodromies around Landau-Ginzburg and conifold points.

\footnotetext{
${ }^{1}$ We do not distinguish defects between non-isomorphic CFTs, by calling them "interfaces" as is, e.g., done in [1].
} 
By means of target space geometric methods those functors have been derived in [13-16] following a proposal by Kontsevich [17]. Using the large volume realisation of B-branes, they can be represented in terms FourierMukai transformations on the bounded derived category of coherent sheaves on the target space manifold, $D^{b}(X)$.

The approach we will take in this article is more direct. We propose to use world-sheet techniques to analyse the behaviour of B-type D-branes, i.e., B-type boundary conditions, under deformations of the underlying bulk conformal field theories along closed loops in its Kähler moduli spaces. This will be studied using a novel method put forward in [12]. Namely, on general grounds one expects that the effect of Kähler perturbations of bulk conformal field theories on B-branes can be described by the fusion with associated B-type supersymmetry preserving defects between the deformed and the undeformed theories.

Thus, our task is to find the specific B-type defects associated to deformations along given loops around singularities in Kähler moduli spaces. As base points for these loops we choose the Landau-Ginzburg or Gepner points which exist for the models we will consider. At these points use can be made of an elegant description of B-branes and B-type defects in terms of matrix factorizations $[11,18-20]$.

To compare the resulting defects to the Fourier-Mukai transformations obtained using target space geometric methods, we need to transport them from the Landau-Ginzburg point in Kähler moduli space to the large volume limit. The functor realizing this transport on the level of B-type Dbranes has been constructed in [21] and has recently been given a physical understanding in [22] (see also [23]). In the latter work, B-type D-branes in gauged linear $\sigma$-models have been defined and studied. This provides a uniform description of B-branes on those parts of Kähler moduli space on which the non-linear $\sigma$-models admit a gauged linear $\sigma$-model realization. In particular, the techniques developed in [22] can be used to transport Btype branes inside these parts of Kähler moduli space, and they immediately generalize to the treatment of B-type supersymmetry preserving defects.

We therefore restrict our considerations to non-linear $\sigma$-models on projective hypersurfaces, which can be realized as gauged linear $\sigma$-models. B-type defects defined at the Landau-Ginzburg point can then be lifted to the gauged linear $\sigma$-model and transported to the large volume limit. As a general result we find that any B-type defect transported from the Landau-Ginzburg point to large volume in this way gives rise to a FourierMukai transformation on the large volume realization $D^{b}(X)$ of the B-brane category. 
Such a relation is not completely unexpected. After all, the "folding trick" allows one to interpret defects between two conformal field theories as boundary conditions in the tensor product of the first of these theories with that (parity dual) of the other one $[3,24]$, and also Fourier-Mukai transformations between two large volume B-brane cateogries are specified by B-branes on the product of the respective target space manifolds.

The Fourier-Mukai transformations that arise by transporting the monodromy defects we construct at the Landau-Ginzburg point to large volume indeed agree with the ones obtained by more geometric methods in $[13,14,16]$.

In fact, lifting defects to the gauged linear $\sigma$-models and transporting them in the respective Kähler moduli spaces provides an independent way to derive the defects describing monodromies generated by loops in the those parts of Kähler moduli space which are covered by a GLSM description. Namely, by lifting the identity defect to the gauged linear $\sigma$-model, transporting it around a singularity in the Kähler moduli space and then pushing it down to the non-linear $\sigma$-model again, one obtains the respective monodromy defect. This will be used as a confirmation of our more general construction of monodromy defects at the Landau-Ginzburg point.

The plan of this paper is as follows: In the remaining part of this Introduction, we review the monodromies in Kähler moduli space of one-parameter models in Section 1.1 and give some details about the fusion of supersymmetric defects in Section 1.2. Following [12], we will motivate that the effect Kähler deformations of bulk theories have on B-branes can be described by the fusion with B-type defects. In Section 2 we review the matrix factorization formalism used to describe B-type D-branes and defects at Landau-Ginzburg points. The monodromy defects will be constructed in this framework. In Section 3 we outline how B-type D-branes and defects can be transported on Kähler moduli space by lifting them to the gauged linear $\sigma$-model. We only give a brief sketch of the constructions we need and illustrate them in the example of tensor product B-branes. For detailed explanations we refer the reader to [22]. Using these techniques, we argue in Section 3.5 that upon transport from the Landau-Ginzburg point, B-type defects generally give rise to Fourier-Mukai transformations at large volume. Section 4 is devoted to the construction and the analysis of the defects corresponding to the monodromy around Landau-Ginzburg points. At the Landau-Ginzburg models themselves, they are obtained as those defects realizing the quantum symmetries which these models exhibit. Transporting them to large volume, we determine the respective Fourier-Mukai kernels. Furthermore, transporting the identity defect around closed loops in Kähler moduli space we also obtain the defects and Fourier-Mukai transformations 
for monodromies around those singularities which can be encircled in the Kähler moduli space of the corresponding gauged linear $\sigma$-model. In Section 5 we construct general conifold monodromies in Landau-Ginzburg models and transport them to large volume. Finally, in Section 6 we conclude with some remarks about directions of future research.

\subsection{D-brane monodromies in Kähler moduli space}

The aim of this paper is to study the dependence of B-type D-branes on Kähler parameters using world-sheet methods. In particular, we are interested in monodromies of B-type D-branes around singular points in the Kähler moduli space. We will focus our considerations on non-linear $\sigma$-models with Calabi-Yau target spaces, which can be realized as hypersurfaces in projective space such as the cubic torus in $\mathbb{P}^{2}$ or the quintic hypersurface in $\mathbb{P}^{4}$.

Such non-linear $\sigma$-models admit a realization as gauged linear $\sigma$-models with U(1) gauge group [25]. The corresponding Kähler moduli space ${ }^{2}$ is given by a two sphere which, however, has three singular points. The large volume point is the limiting point where the volume of the target space goes to infinity. Similarly, at the conifold point the (quantum) volume of the target space goes to zero. Finally, the Landau-Ginzburg or Gepner point is an orbifold point in the Kähler moduli space. At this point certain dualities of the theories under consideration become self-dualities and enhance the symmetry group of the theory.

We would like to study what happens to B-type D-branes when one moves around these singular points in Kähler moduli space. As alluded to above, from a world-sheet point of view this means that one has to understand how boundary conditions evolve under deformations of the bulk conformal field theories.

For a general CFT however, boundary conditions are very difficult to construct. Even worse, in the situations we are interested in, the CFTs themselves are only known explicitly at special points in the Kähler moduli spaces and conformal perturbation theory becomes intractable at higher orders in the perturbation parameters.

\footnotetext{
${ }^{2}$ In general, the Kähler moduli spaces of the gauged linear $\sigma$-models only capture twodimensional submanifolds of the non-linear $\sigma$-model Kähler moduli spaces. In case of projective hypersurfaces of dimension three and higher the Lefschetz hyperplane theorem guarantees that the two Kähler moduli spaces coincide.
} 
Fortunately, there are relatively nice descriptions of B-type branes at special points in the Kähler moduli space. At large volume for instance Btype D-branes have a geometric realization in terms of bounded complexes of coherent sheaves on the target space [26-28], and as mentioned above, using target space geometric arguments one can determine their monodromies around the singular points in Kähler moduli space.

Namely, it has been been shown how the conifold monodromy manifests itself as a result of stability considerations in [14]. The basic physical idea underlying these arguments is that at a conifold point the quantum worldvolume of a particular D-brane collapses to zero. The D-brane becomes massless [29] and its central charge goes to zero at this point. Therefore, this D-branes can be created at the conifold point at no cost in energy. The quantum world-volume of other D-branes and therefore their masses remain finite. If such a massive probe brane is transported around the conifold point, the open string states between the probe brane and the D-brane which is massless at the conifold point become tachyonic at some point, triggering a tachyon condensation of these D-branes to an energetically preferable bound state. Thus, taking the probe brane around the conifold point, one ends up with a bound state of it with the D-brane which becomes massless at the conifold point $[14,30,31]$.

Moreover, it is not difficult to see that the large volume monodromy can be understood in terms of a shift in the B-field $B \rightarrow B+\xi$, where $\xi$ is the generator ${ }^{3}$ of $H^{1,1}(X)$. Since the B-field enters the relevant charge formulas for large volume B-branes only in the combination $B+F$ with the field strength $F$ of the U(1)-connection on the D-brane, the action of the monodromy is given by tensoring the Chan-Paton bundle of the B-brane with a line bundle of first Chern class $c_{1}=[\xi]^{4}$.

The monodromy around the Landau-Ginzburg point is not so easy to derive directly by means of geometric arguments. However, it can be obtained by composing conifold and large volume monodromies. A more direct derivation of this monodromy appeared in [33], combining linear $\sigma$-model methods for the open string with the fact that this monodromy is given by the quantum symmetry at the Landau-Ginzburg point.

\footnotetext{
${ }^{3}$ If $\operatorname{dim} H^{1,1}(X)>1$ we will only consider the monodromy around the large volume limit in the slice of the moduli space covered by the GLSM construction. Then $\xi$ is the generator of $H^{1,1}(X)$ inherited from the one-dimensional cohomology group $H^{2}\left(\mathbb{P}^{N-1}, \mathbb{R}\right)$ of the embedding projective space.

${ }^{4}$ Note that the appearance of $F+B$ in the charge formulas a priori justifies the action of the monodromy on the level of K-theory. However, by studying B-type D-branes in the context of open topological string field theory [27,32], this action carries over to the derived category.
} 
To derive these monodromies directly using world-sheet techniques, one has to deform the bulk conformal field theory along loops in Kähler moduli space and analyse how B-type boundary conditions behave under these deformations. The straightforward way to approach this problem is to use conformal perturbation theory, which however becomes intractable at higher orders. Therefore we will employ a method put forward in [12] to analyse the effect of bulk perturbations on boundary conditions. Namely, we will use the fact that Kähler perturbations can be described by B-type defects between the unperturbed and the perturbed theory. This will be explained in more detail in Section 1.2 below.

\subsection{Bulk perturbations and defects}

In this section we will briefly outline some aspects of defects and their fusion. Furthermore, we will motivate why the effect of bulk perturbations of conformal field theories on boundary conditions can be described by the fusion with defects between the unperturbed and the perturbed theory.

As mentioned above, defects are one-dimensional junctions separating two possibly different conformal field theories on the same world-sheet (cf. figure 1).

Two such defects between conformal field theories can be brought on top of each other to form a new defect as illustrated in figure 2. Such a "fusion" process involves taking the limit in which the distance between the defects shrinks to zero. On the level of the full conformal field theory this process is highly singular in general and needs to be regularized (see [1] for an explicit calculation in the example of the free boson CFT). In case

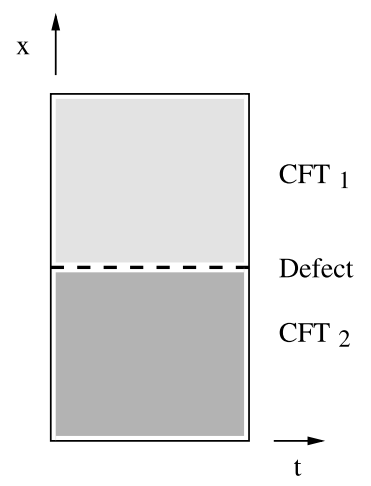

Figure 1: Two two-dimensional CFTs are separated by a one-dimensional defect. 


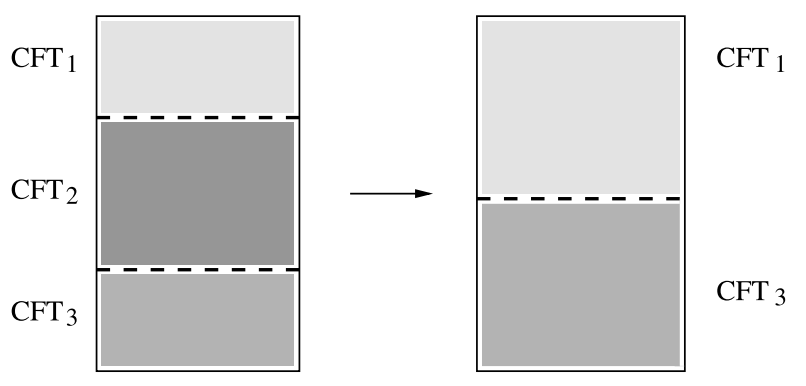

Figure 2: In the limit where the two defects coincide, a new defect connecting theories 1 and 3 emerges.

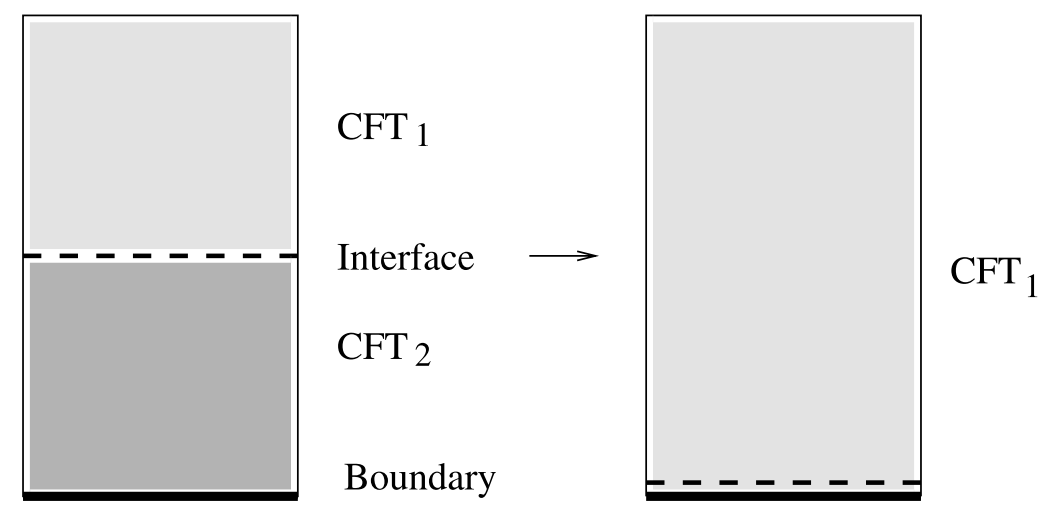

Figure 3: In the limit where the defect coincides with the boundary, a new boundary condition of theory 1 emerges.

the underlying conformal field theories are $N=2$ superconformal, and the defects preserve the same $N=2$ superconformal symmetry, this fusion can be regularized. Indeed, it can be described on the level of the topologically twisted theory, where correlators do not depend on positions on the worldsheet, in particular not on those of defects. Hence, in the topologically twisted theory, correlators do not exhibit singularities when defects approach each other, and fusion is well defined.

In the same way, defects can be brought on top of world-sheet boundaries to transform the original boundary conditions imposed there into new ones (cf. figure 3). In general, also the fusion of a defect and a boundary condition is singular, but as in the case of fusion of two defects, it can be regularized if defect and boundary conditions preserve the same $N=2$ superconformal symmetry. (In fact, boundary conditions in a conformal field theory can be regarded as defects between this CFT and the trivial one, so that boundary conditions are in fact a special type of defects.) 
In this way, fusion endows the set of all defects preserving a specific $N=2$ superconformal symmetry with a multiplicative structure, and these defects act on the category of D-branes preserving the same $N=2$ algebra by means of functors between the respective D-brane categories. For the case of Landau-Ginzburg theories, fusion of B-type defects and their action on boundary conditions has been investigated in $[11,12]$.

Analogously to D-branes, also defects exhibit fields which are defined on them. These "defect-changing fields" can be used to perturb defects in the same way as "boundary condition-changing fields" can be used to perturb boundary conditions. In particular, also defects can undergo tachyon condensation and bound state formation. Not surprisingly, such bound states will figure prominently in the construction of defects describing monodromies around conifold points.

Defects have a rich structure and are very interesting objects, and they also turn out to be very useful. For instance, it has been realized in [12] that they can be employed to study the behaviour of D-branes under (marginal or relevant) perturbations of the underlying bulk conformal field theory. ${ }^{5}$

The basic reasoning behind this is to restrict the perturbation of a conformal field theory on a world-sheet with boundary a fixed distance $\epsilon$ away from the latter. Performing the renormalization group flow associated to this restricted perturbation, one ends up with the following situation in the IR (cf. figure 4). On the part of the surface the perturbation was restricted to, the theory has flown from the UV to the IR of the particular bulk perturbation considered, whereas the theory in the $\epsilon$-strip around the boundary from which the perturbation was kept away stays at the UV. These two theories are separated by a defect line situated in $\epsilon$-distance from the boundary. In this way, every bulk perturbation gives rise to a unique "flow defect."

Moving this defect towards the boundary, i.e., sending $\epsilon$ to zero, spreads the perturbation to the entire surface. As alluded to above this process is singular in general. That is not surprising, because the perturbing bulk fields can have singularities when they approach the boundary. For that reason the unrestricted bulk perturbation on the surface with boundary needs to be regularized and counterterms on the boundary have to be added. In general, such an unrestricted bulk perturbation will therefore also trigger a simultaneous boundary perturbation. In the same way, the process of taking the defect towards the boundary needs to be regularized. The regularized fusion of the defect with the boundary condition then describes to what boundary condition in the IR theory a given boundary condition in the UV

\footnotetext{
${ }^{5}$ Boundary flows have been realized by means of defects in $[5,8]$.
} 


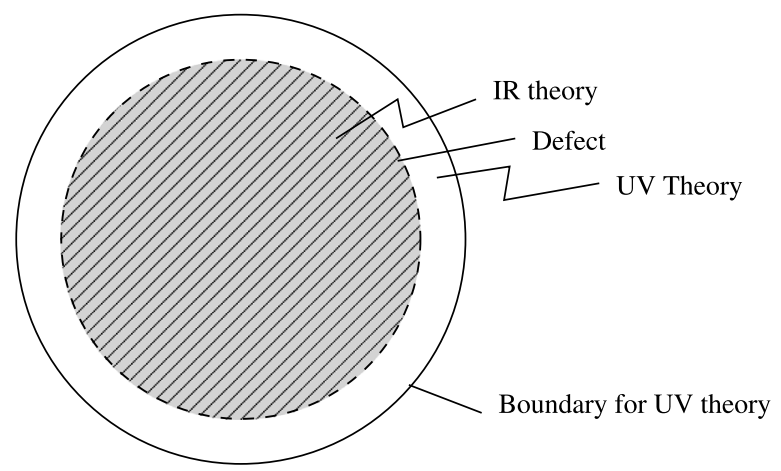

Figure 4: The perturbation is restricted to the shaded domain. UV and IR are separated by a defect. Fusing this defects with the UV boundary, one obtains the IR boundary condition.

theory flows under the bulk perturbation, i.e., it encodes the behaviour of boundary conditions under bulk flows.

The corresponding regularization can be very tricky however. Fortunately, we are interested in a special case, namely bulk perturbations of $N=(2,2)$ superconformal field theories, which preserve an $N=2$ supersymmetry even in the presence of world-sheet boundaries. For these perturbations, one can topologically twist the corresponding theories all along the flow. The flow defects also preserve the $N=2$ supersymmetry in this case, and its fusion with boundary conditions can be effectively described in the twisted theory, where no singularities arise when the defect is moved towards the boundary. In this way, it is possible to avoid dealing with regularization issues for these particular perturbations.

The condition that the perturbation preserves supersymmetry on a surface with boundary however is very constraining. There are two classes of $N=(2,2)$ supersymmetry preserving bulk perturbations, those generated by elements of the $(c, c)$ - and ( $a, a)$-rings (chiral multiplets) which correspond to "complex structure-type" perturbations, and those generated by elements of the $(a, c)$ - and $(c, a)$-rings (twisted-chiral multiplets), which correspond to "Kähler-type" perturbations. Concretely, the bulk perturbation generated by a twisted chiral field takes the form (notations are taken from [34])

$$
\Delta S=\left.\int d \bar{\theta}^{-} d \theta^{+} \Psi\right|_{\bar{\theta}^{+}=\theta^{-}=0}+\text { c.c. },
$$

where $\Psi$ is the perturbing twisted chiral multiplet. On world-sheets without boundary, the perturbation is invariant under the full $N=(2,2)$ - 
supersymmetry variation

$$
\delta=\epsilon_{+} Q_{-}-\epsilon_{-} Q_{+}-\bar{\epsilon}_{+} \bar{Q}_{-}+\bar{\epsilon}_{-} \bar{Q}_{+}
$$

On world-sheets with boundaries one can impose A- or B-type boundary conditions and preserve one half of the supersymmetry. An A-type supersymmetry variation (setting $\epsilon:=\epsilon_{+}=\bar{\epsilon}_{-}$in (1.2)) of a twisted chiral perturbation yields a boundary term that in general cannot be cancelled by the supersymmetry variation of a boundary term. ${ }^{6}$ Hence, in the presence of a boundary on which an A-type boundary condition is imposed, the bulk perturbation generated by a twisted chiral field breaks supersymmetry.

On the other hand, the boundary term coming from a B-type supersymmetry variation (i.e., $\epsilon:=\epsilon_{+}=-\epsilon_{-}, \bar{\epsilon}:=\bar{\epsilon}_{+}=-\bar{\epsilon}_{-}$) can always be cancelled by adding a simple additional boundary term involving only bulk fields

$$
\mathcal{B} \sim \int_{\partial \Sigma}(\psi-\bar{\psi})
$$

where $\psi$ denotes the upper component of the twisted chiral superfield $\Psi$. Thus, twisted chiral bulk perturbations preserve supersymmetry in the presence of boundaries with B-type boundary conditions. ${ }^{7}$

Hence, it can be concluded that Kähler-type perturbations preserve supersymmetry in the presence of boundary conditions of B-type but not in general of those of A-type. The flow defects associated to these perturbations preserve the same B-type supersymmetry. Similarly, complex structure-type perturbations preserve supersymmetry in the presence of boundary conditions of A-type but not of those of B-type, and the corresponding flow defects preserve A-type supersymmetry.

In both these cases regularization issues in the fusion of flow defects with boundary conditions can be avoided by considering the fusion in the topologically twisted theory, the B-twisted one in the first case, and the A-twisted one in the second.

\footnotetext{
${ }^{6}$ In fact, this calculation is mirror to the one performed in $[18,19,34]$ in the case of B-type boundary conditions for the superpotential part of the action. There, additional degrees of freedom at the boundary helped to cancel the undesired term. Here, we can only use the fields that are already part of the theory.

${ }^{7}$ Note however that the perturbations can rotate the gluing conditions imposed on the spectral flow operator. On the level of CFT this can be seen using the methods of [35]. From a space time point of view, this implies that the target space supersymmetry is rotated and possibly broken. These effects were fundamental for the discussion of П-stability.
} 
In this article we are interested in the first of these two cases. Namely, from a world-sheet perspective, monodromies of B-branes in the Kähler moduli space of non-linear $\sigma$-models are obtained by deforming the non-linear $\sigma$-model in the presence of B-type boundary conditions along closed loops in Kähler moduli space. By the reasoning above the effect of this deformation on B-branes can be described by the fusion with a B-type flow-defect.

\section{B-type defects in Landau-Ginzburg models and matrix factorizations}

In Landau-Ginzburg models, both B-type supersymmetric D-branes and B-type supersymmetry preserving defects have an elegant description in terms of matrix factorizations [11,18-20]. A matrix factorization $P$ of a polynomial $W \in \mathbb{C}\left[x_{1}, \ldots, x_{N}\right]$ is given by a pair $\left(P_{1}, P_{0}\right)$ of free $\mathbb{C}\left[x_{1}, \ldots, x_{N}\right]$ modules together with homomorphisms $p_{s}: P_{s} \rightarrow P_{(s+1) \bmod 2}$ between them which compose to $W$ times the identity map, i.e., $p_{1} p_{0}=W \operatorname{id}_{P_{0}}$ and $p_{0} p_{1}=$ $W \operatorname{id}_{P_{1}}$. In the following we will often represent matrix factorizations by

$$
P: P_{1} \underset{p_{0}}{\stackrel{p_{1}}{\rightleftarrows}} P_{0} .
$$

Sometimes it is useful to regard them as two-periodic twisted ${ }^{8}$ complexes. Indeed, such matrix factorizations form a category, with morphisms $\mathcal{H}^{*}(P, Q)$ between two matrix factorizations $P$ and $Q$ given by the cohomology of the Hom-complex of the two twisted complexes associated to $P$ and $Q$. The latter is a two-periodic untwisted complex.

There are always matrix factorizations with modules $P_{s}=\mathbb{C}\left[x_{1}, \ldots, x_{N}\right]$ where one of the maps $p_{r}=1$ and the other one $p_{(r+1) \bmod 2}=W$. They are trivial in that they only have zero-morphisms with any other (including themselves) matrix factorization, and two matrix factorizations which differ by the addition of such a trivial matrix factorization are equivalent.

As was shown in $[18,19]$, B-type supersymmetric D-branes in LandauGinzburg models with chiral superfields $x_{1}, \ldots, x_{N}$ and superpotential $W \in$ $\mathbb{C}\left[x_{1}, \ldots, x_{N}\right]$ can be represented by matrix factorizations of $W$, where open strings between two such D-branes are described by morphisms between the respective matrix factorizations.

In the same way, it has been argued in [11] that B-type supersymmetry preserving defects between two Landau-Ginzburg models, one with chiral

\footnotetext{
${ }^{8}$ The differential squares to $W$.
} 
fields $x_{1}, \ldots, x_{N}$ and superpotential $W_{1} \in \mathbb{C}\left[x_{1}, \ldots, x_{N}\right]$ and one with chiral superfields $y_{1}, \ldots, y_{M}$ and superpotential $W_{2} \in \mathbb{C}\left[y_{1}, \ldots, y_{M}\right]$ can be represented by matrix factorizations of $W_{1}-W_{2}$ over the polynomial ring $\mathbb{C}\left[x_{1}, \ldots, x_{N}, y_{1}, \ldots, y_{N}\right]$.

As mentioned in Section 1.2, one interesting property of $N=2$ supersymmetric defects is that they can be fused with other such defects, or with D-branes (boundary conditions) preserving the same supersymmetry. Namely, two such defects can be brought on top of each other to produce a new defect, or a defect can be moved onto a world sheet boundary to change the boundary condition imposed there. This fusion has a very simple realization in terms of the matrix factorization description. For instance, let $x_{i}, y_{i}, z_{i}$ be the chiral superfields of three Landau-Ginzburg models with superpotentials $W_{1} \in \mathbb{C}\left[x_{i}\right], W_{2} \in \mathbb{C}\left[y_{i}\right]$ and $W_{3} \in \mathbb{C}\left[z_{i}\right]$, respectively, which are separated by two defects represented by matrix factorizations $P^{1}$ of $W_{1}-W_{2}$ and $P^{2}$ of $W_{2}-W_{3}$. Fusing the two defects gives rise to a new defect separating the Landau-Ginzburg model with chiral fields $x_{i}$ and superpotential $W_{1}$ from the one with chiral fields $z_{i}$ and superpotential $W_{3}$. This fused defect is given by the matrix factorization

$$
P^{1} * P^{2}=\left(P^{1} \otimes P^{2}\right)_{\mathbb{C}\left[x_{i}, z_{i}\right]}^{\mathrm{red}} .
$$

Here, the tensor product of two matrix factorizations is defined by taking the tensor product of the associated twisted complexes, which again is a two-periodic complex which is twisted by the sum of the twists of the tensor factors. More concretely, the tensor product $P \otimes Q$ of matrix factorizations $P$ and $Q$ of $W$ and $W^{\prime}$, respectively, can be written as

$$
P \otimes Q: P_{1} \otimes Q_{0} \oplus P_{0} \otimes Q_{1} \underset{r_{0}}{\stackrel{r_{1}}{\rightleftarrows}} P_{0} \otimes Q_{0} \oplus P_{1} \otimes Q_{1}
$$

with

$$
r_{1}=\left(\begin{array}{cc}
p_{1} \otimes \mathrm{id} & \mathrm{id} \otimes q_{1} \\
-\mathrm{id} \otimes q_{0} & p_{0} \otimes \mathrm{id}
\end{array}\right), \quad r_{0}=\left(\begin{array}{cc}
p_{0} \otimes \mathrm{id} & -\mathrm{id} \otimes q_{1} \\
\mathrm{id} \otimes q_{0} & p_{1} \otimes \mathrm{id}
\end{array}\right),
$$

which is a matrix factorization of $W+W^{\prime}$.

In the situation above, $P^{1}$ is a matrix factorization of $W_{1}-W_{2}$ and $P^{2}$ one of $W_{2}-W_{3}$. Hence, $P^{1} \otimes P^{2}$ is a matrix factorization of $W_{1}-W_{3} \in$ $\mathbb{C}\left[x_{i}, z_{i}\right]$, but it is still a matrix factorization over $\mathbb{C}\left[x_{i}, y_{i}, z_{i}\right]$. That means that the modules $\left(P^{1} \otimes P^{2}\right)_{s}$ are free $\mathbb{C}\left[x_{i}, y_{i}, z_{i}\right]$-modules and also the maps $r_{s}$ between them depend on the $y_{i}$. The notation $\left(P^{1} \otimes P^{2}\right)_{\mathbb{C}\left[x_{i}, z_{i}\right]}$ means that this matrix factorization has to be regarded as one over $\mathbb{C}\left[x_{i}, z_{i}\right]$ only. 
As such, it is of infinite rank, because the modules $\left(P^{1} \otimes P^{2}\right)_{s}$ regarded as modules over $\mathbb{C}\left[x_{i}, z_{i}\right]$ are free modules of infinite rank. For instance, $\mathbb{C}\left[x_{i}, y_{i}, z_{i}\right]$ can be decomposed as

$$
\mathbb{C}\left[x_{i}, y_{i}, z_{i}\right]=\bigoplus_{\left(l_{1}, \ldots, l_{N}\right) \in \mathbb{N}_{0}^{N}} y_{1}^{l_{1}} \ldots y_{N}^{l_{N}} \mathbb{C}\left[x_{i}, z_{i}\right]
$$

into free $\mathbb{C}\left[x_{i}, z_{i}\right]$-modules. Physically speaking, the chiral fields $y_{i}$ of the theory squeezed in between the two defects are promoted to new defect degrees of freedom in the limit where the two defects coincide. However, most of them are trivial. Namely, if both $P^{1}$ and $P^{2}$ are of finite rank, the matrix factorization $\left(P^{1} \otimes P^{2}\right)_{\mathbb{C}\left[x_{i}, z_{i}\right]}$ can be reduced to finite rank by splitting off infinitely many trivial matrix factorizations. It is the result of this reduction $\left(P^{1} \otimes P^{2}\right)_{\mathbb{C}\left[x_{i}, z_{i}\right]}^{\mathrm{red}}$ which describes the fused defect. More details about this can be found in [11].

In the same way, fusion of B-type defects and B-type D-branes in LandauGinzburg models can be formulated in the matrix factorization framework. The fusion of a B-type defect separating a Landau-Ginzburg model with chiral fields $x_{i}$ and superpotential $W_{1} \in \mathbb{C}\left[x_{i}\right]$ from one with chiral fields $y_{i}$ and superpotential $W_{2} \in \mathbb{C}\left[y_{i}\right]$ and a B-type D-brane (boundary condition) in the second of these Landau-Ginzburg models can be represented by the matrix factorization

$$
P * Q=(P \otimes Q)_{\mathbb{C}\left[x_{i}\right]}^{\mathrm{red}},
$$

where $P$ is the matrix factorization of $W_{1}-W_{2}$ associated to the defect and $Q$ the matrix factorization of $W_{2}$ associated to the D-brane.

This formalism can be easily generalized to the context of LandauGinzburg orbifolds. If $\Gamma$ is an orbifold group in a Landau-Ginzburg model with chiral fields $x_{1}, \ldots, x_{N}$ and superpotential $W \in \mathbb{C}\left[x_{1}, \ldots, x_{N}\right]$, then it has an action $\rho: \Gamma \rightarrow \operatorname{End}\left(\mathbb{C}\left[x_{1}, \ldots, x_{N}\right]\right)$ on $\mathbb{C}\left[x_{1}, \ldots, x_{N}\right]$ which is compatible with multiplication, i.e.,

$$
\rho(\gamma)(a b)=\rho(\gamma)(a) \rho(\gamma)(b) \quad \text { for } a, b \in \mathbb{C}\left[x_{1}, \ldots, x_{N}\right], \gamma \in \Gamma,
$$

and which leaves $W$ invariant. The Landau-Ginzburg orbifolds which will appear in this article are of the following type. $W \in \mathbb{C}\left[x_{1}, \ldots, x_{N}\right]$ is a homogeneous polynomial of degree $d$, and the orbifold group $\Gamma=\mathbb{Z}_{d}$ acts on 
monomials of degree $l$ by phase multiplications:

$$
\gamma(n)\left(x_{1}^{l_{1}} \ldots x_{N}^{l_{N}}\right)=e^{\frac{2 \pi i}{d} n \sum_{i} l_{i}} x_{1}^{l_{1}} \ldots x_{N}^{l_{N}} \quad n \in \mathbb{Z}_{d}
$$

B-type D-branes in Landau-Ginzburg orbifolds can then be represented by $\Gamma$-equivariant matrix factorizations of $W[36,37]$. These are matrix factorizations

$$
P: P_{1} \underset{p_{0}}{\stackrel{p_{1}}{\rightleftarrows}} P_{0}
$$

together with representations $\rho_{s}$ of $\Gamma$ on $P_{s}$, which are compatible with the module structure

$$
\rho_{s}(\gamma)(a r)=\rho(\gamma)(a) \rho_{s}(\gamma)(r) \quad \text { for } a \in \mathbb{C}\left[x_{1}, \ldots, x_{N}\right], \gamma \in \Gamma, r \in P_{s}
$$

and commute with the maps $p_{s}$

$$
\rho_{(s+1) \bmod 2}(\gamma) p_{s}=p_{s} \rho_{s}(\gamma) \quad \text { for } \gamma \in \Gamma, s=0,1
$$

Note that because of the compatibility with the multiplication, the $\rho_{i}$ are fixed by the respective action on the degree-zero subspaces $\mathbb{C}^{k} \subset \mathbb{C}\left[x_{i}\right]^{k} \cong$ $P_{s}$. It is this action which we will later specify in the definition of equivariant matrix factorizations. In the case of $\mathbb{Z}_{d^{-}}$or $\mathrm{U}(1)$-actions we will often indicate the respective charge $q$ of $1 \in \mathbb{C}\left[x_{i}\right]$ by writing $\mathbb{C}\left[x_{i}\right][q]$.

Given two such equivariant matrix factorizations $P$ and $Q$ the above conditions ensure that the $\Gamma$-action pushes through to the cohomology of the Hom-complex, so that one can define the space of morphism in the category of equivariant matrix factorizations to be $\mathcal{H}_{\text {orb }}^{*}(P, Q)=\left(\mathcal{H}^{*}(P, Q)\right)^{\Gamma}$, the $\Gamma$ invariant part of the morphism spaces in the underlying unorbifolded category. These are the spaces of open strings between the respective D-branes in the orbifold Landau-Ginzburg model.

Similarly, B-type defects separating a Landau-Ginzburg orbifold with chiral fields $x_{i}$, superpotential $W_{1} \in \mathbb{C}\left[x_{i}\right]$ and orbifold group $\Gamma_{1}$ and one with chiral fields $y_{i}$, superpotential $W_{2} \in \mathbb{C}\left[y_{i}\right]$ and orbifold group $\Gamma_{2}$ can be represented by $\Gamma_{1} \times \Gamma_{2}$-equivariant matrix factorizations of $W_{1}-W_{2}$ [12]. The conditions on the $\Gamma$-representations above imply that the matrix factorization obtained by fusing two equivariant matrix factorizations $P$ and $Q$ in the unorbifolded category is again equivariant with respect to the product of all orbifold groups present. In particular, one can take the part of this matrix factorization which is invariant under the orbifold group $\Gamma_{\text {squeezed }}$ of the model which is squeezed in between the two defects, or the defect and 
the boundary. This represents the fusion in the orbifold theory

$$
P *_{\text {orb }} Q=(P * Q)^{\Gamma_{\text {squeezed }}} .
$$

Since most of the discussion in the following will be concerned with orbifolds of Landau-Ginzburg models, we will often omit to write the subscript "orb" in case confusion is unlikely.

As alluded to above, in this article we are dealing with homogeneous superpotentials $W$ of degree $d$. The corresponding Landau-Ginzburg models exhibit a $\mathrm{U}(1)_{R^{-}}$-symmetry which acts on the chiral fields as

$$
\rho^{R}(\varphi)\left(x_{1}^{l_{1}} \ldots x_{N}^{l_{N}}\right)=\mathrm{e}^{2 \pi \mathrm{i} \varphi \sum_{i} \frac{2 l_{i}}{d}} x_{1}^{l_{1}} \ldots x_{N}^{l_{N}} \quad \varphi \in \mathbb{R},
$$

i.e., the $x_{i}$ have $\mathrm{U}(1)_{R}$-charge $\frac{2}{d}$, such that the superpotential has charge 2 . The existence of this additional symmetry guarantees that the theory flows to a conformal field theory in the IR. Similarly, also B-type boundary conditions and defects of the Landau-Ginzburg model flow to conformal boundary conditions and defects in the IR if they preserve this $\mathrm{U}(1)_{R}$-symmetry. This means that they are given by $\mathrm{U}(1)_{R}$-equivariant matrix factorizations. Since $W$ is not invariant under $\mathrm{U}(1)_{R}$, but has $R$-charge 2 , one has to slightly modify the definition of equivariant matrix factorizations in this case. More precisely the commutation relation (2.11) cannot hold in this case, but it has to be replaced by

$$
\rho_{(s+1) \bmod 2}^{R}\left(\mathrm{e}^{2 \pi \mathrm{i} \varphi}\right) p_{s}=\mathrm{e}^{2 \pi \mathrm{i} \varphi} p_{s} \rho_{s}^{R}\left(\mathrm{e}^{2 \pi i \varphi}\right),
$$

reflecting the fact that the maps $p_{s}$ have $R$-charge 1 . Note that there is a relation between the representation $\rho^{R}$ of $\mathrm{U}(1)_{R}$ and the representation $\rho$ of the orbifold group $\Gamma=\mathbb{Z}_{d}$. First of all the representation of $\mathrm{U}(1)_{R}$ and $\Gamma=\mathbb{Z}_{d}$ commute with each other. Secondly, the combination $\rho^{R}\left(\frac{1}{2}\right) \rho(-1)$ leaves all chiral fields $x_{i}$ invariant. Thus, for irreducible matrix factorizations $P, \rho_{s}^{R}\left(\frac{1}{2}\right) \rho_{s}(-1) \sim \operatorname{id}_{P_{s}}$. Furthermore, $\rho_{s}^{R}\left(\frac{1}{2}\right) \rho_{s}(-1)$ anti-commute with the maps $p_{s}$, and it can be chosen to be

$$
\rho_{s}^{R}\left(\frac{1}{2}\right) \rho_{s}(-1)=(-1)^{s} \operatorname{id}_{P_{s}} .
$$

Diagonalizing the actions of $\rho_{s}^{R}$ and $\rho_{s}$ simultaneously, one arrives at the following relation between the $\mathrm{U}(1)_{R}$-charges $r$ and $\Gamma=\mathbb{Z}_{d^{-}}$charges $q$ on the modules $P_{s}$

$$
r-\frac{2 q}{d} \in 2 \mathbb{Z}+s \text { on } P_{s} .
$$




\section{Gauged linear sigma models and matrix factorizations}

To compare the monodromies we obtain in terms of defects at the LandauGinzburg points with the monodromy transformations at large volume derived by more geometric methods $[16,30]$, we need to transport B-type Dbranes between these two points in Kähler moduli space. The corresponding functors between the categories of B-branes in Landau-Ginzburg models and those in non-linear $\sigma$-models has been constructed in [21]. A physical understanding of these functors has been developed in [22], using a gauged linear $\sigma$-model description of B-type D-branes, see $[23,33,38,39]$ for earlier work.

Indeed, the models we are considering have a realization in terms of gauged linear $\sigma$-models with gauge group $\mathrm{U}(1)$, chiral superfields $\left(\mathfrak{p}, x_{1}, \ldots\right.$, $\left.x_{N}\right)$ of $\mathrm{U}(1)$-charge $(-d, 1, \ldots, 1)$ and $R$-charge $(2,0, \ldots, 0)$, and superpotential $\mathfrak{p} W\left(x_{1}, \ldots, x_{N}\right)$, where $W$ is a polynomial of degree $d$ in the fields $x_{i}$ (see [25]). We are mostly interested in the Calabi-Yau case, in which $d=N$. These models depend on a complex parameter $t=r+i \theta$, a combination of the Fayet-Iliopoulus parameter $r$ and the $\theta$-angle, which parametrizes their Kähler moduli spaces $(r, \theta) \in(\mathbb{R} \times \mathbb{R} / 2 \pi \mathbb{Z}) \backslash\{(d \log d,[\pi d])\}$. The large volume limit point is situated at $t=\infty$, where the GLSM reduces to a $\sigma$-model on the projective hypersurface $X \subset \mathbb{P}^{N-1}$ defined by $W=0$. At $t=-\infty$ on the other hand the field $\mathfrak{p}$ receives a non-vanishing vacuum expectation value, which breaks the gauge group down to $\mathbb{Z}_{d}$, and the GLSM reduces to a Landau-Ginzburg orbifold with chiral superfields $x_{i}$, superpotential $W$ and orbifold group $\Gamma=\mathbb{Z}_{d}$. The conifold point corresponds to $(r=d \log d, \theta=[\pi d])$.

The GLSMs provide a uniform description of all the models in Kähler moduli space. A description of D-branes in these models therefore allows to transport B-type D-branes between arbitrary points in Kähler moduli space. This has been worked out in [22], and will be used here to compare monodromies at Landau-Ginzburg and large volume points. In this section we will briefly describe the techniques we need. For more details we refer the reader to $[22]$.

\subsection{B-type D-branes in GLSMs}

Because of the monodromies B-type D-branes exhibit on the Kähler moduli spaces of gauged linear $\sigma$-models, a uniform description of these D-branes can only be expected on a cover of these moduli spaces. In the cases we are considering, this cover is given by $\mathbb{R} \times \mathbb{R} \backslash(\{d \log d\} \times 2 \pi \mathbb{Z}+\pi d)$. On it, B-type D-branes can be represented by $\mathrm{U}(1) \times \mathrm{U}(1)_{R}$-equivariant 
matrix factorizations of $\mathfrak{p} W\left(x_{1}, \ldots, x_{N}\right)$. However, on the phase boundary $r=d \log d$ not all of these matrix factorizations lead to physically welldefined boundary conditions. This gives rise to the "grade restriction rule" of [22]. Namely, on a component $N_{n}=\{d \log d\} \times(\pi d-2 \pi(n+1)+(0,2 \pi))$ of the phase boundary, only matrix factorizations give rise to well-defined boundary conditions, whose $\mathrm{U}(1)$-charges $q$ satisfy

$$
1-d+n \leq q \leq n
$$

We denote the respective "window" of integral U(1)-charges compatible with this phase boundary component $\mathcal{N}_{n}=\{1-d+n, \ldots, n\}$.

Our aim is to transport B-branes from the Landau-Ginzburg to the large volume point of such a GLSM, where they can be represented by matrix factorizations of $W$ and complexes of coherent sheaves on $X$, respectively. To this end, we will discuss in the following how B-branes behave under the reduction of the GLSM to these limiting models. We will, however, only present the recipe here. More detailed explanations can be found in [22].

\subsection{Reduction to Landau-Ginzburg models}

In the Landau-Ginzburg phase of the GLSM the field $\mathfrak{p}$ receives a nonvanishing vacuum expectation value [25], which can be gauged to 1 . This breaks the gauge group from $\mathrm{U}(1)$ down to $\mathbb{Z}_{d}$. The fields $x_{i}$ remain massless and the model reduces to the Landau-Ginzburg orbifold with superpotential $W$ and orbifold group $\Gamma=\mathbb{Z}_{d}$.

On the level of B-type D-branes, a $\mathrm{U}(1) \times \mathrm{U}(1)_{R^{-}}$equivariant matrix factorization $\hat{P}$ representing a B-type brane in the GLSM reduces to a $\mathbb{Z}_{d} \times$ $\mathrm{U}(1)_{R}$-equivariant matrix factorization of $W$, representing a B-type brane in the Landau-Ginzburg orbifold in the following way. One sets all entries $\mathfrak{p}$ in the maps $\hat{p}_{s}$ to 1 and replaces $\hat{P}_{s}$ by $\hat{P}_{s} / \mathfrak{p} \hat{P}_{s}$. This reduces the $\mathrm{U}(1)-$ equivariance to a $\mathbb{Z}_{d}$-equivariance, where the representations $\hat{\rho}_{s}$ are replaced by the induced representations of $\mathbb{Z}_{d} \subset \mathrm{U}(1)$. Furthermore, the $\mathrm{U}(1)_{R^{-}}$ representations $\rho_{s}^{R}$ of the reduced matrix factorizations arise as the combination $\rho_{s}^{R}=\hat{\rho}_{s}^{R} \hat{\rho}_{s}^{2}$ of the $\mathrm{U}(1)_{R}$ and $\mathrm{U}(1)$-representations of the GLSM matrix factorizations.

Since we would like to transport B-type branes from the Landau-Ginzburg to the large volume point, we are interested in the reverse of this reduction. Namely, given a B-brane in the Landau-Ginzburg orbifold, i.e., a $\mathbb{Z}_{d} \times \mathrm{U}(1)_{R}$-equivariant matrix factorization $P$ of $W$, we would like to lift it 
to a $\mathrm{U}(1) \times \mathrm{U}(1)_{R}$-equivariant matrix factorization $\hat{P}$ of $\mathfrak{p} W$, which can be transported through a specified component $N_{n}$ of the phase boundary, and which by the above procedure reduces to $P$ at the Landau-Ginzburg point. Indeed, in the Calabi-Yau case, for every matrix factorization $P$ of $W$ and every component $N_{n}$ of the phase boundary, there is exactly one such lift $\hat{P}[22]$. The lifts can be constructed by introducing factors $\mathfrak{p}$ into the maps $p_{s}$ in such a way that the representations $\rho_{s}$ of $\mathbb{Z}_{d}$ lift to representations $\hat{\rho}_{s}$ of $\mathrm{U}(1)$ and that the corresponding charges lie in the charge window $\mathcal{N}_{n}$. We refrain from giving more details about the general construction here, but will later on explicitly discuss the lifts we will need. In particular, the case of tensor product B-branes is discussed in Section 3.4 below.

\subsection{Reduction to large volume}

At large volume, one recovers the complex of coherent sheaves representing the large volume B-brane out of the GLSM matrix factorization $\hat{P}$ in the following way. Consider the module $\mathcal{P}=\hat{P}_{0} \oplus \hat{P}_{1} / W\left(\hat{P}_{0} \oplus \hat{P}_{1}\right)$ and regard it as $R=\mathbb{C}\left[x_{1}, \ldots, x_{N}\right] /(W)$-module. Because of the presence of the $\mathfrak{p}$, it

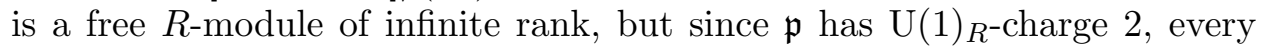
submodule $\mathcal{P}^{r}$ of fixed $\mathrm{U}(1)_{R}$-charge $r \in \mathbb{Z}$ is a free $R$-module of finite rank. Moreover, there is an $r_{\min } \in \mathbb{Z}$, such that for all $r<r_{\min } \mathcal{P}^{r}=0$, and, having

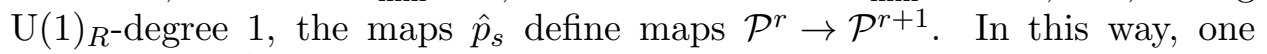
obtains from $\hat{P}$ a $U(1)$-equivariant complex

$$
\mathcal{P}: \mathcal{P}^{r_{\min }} \rightarrow \mathcal{P}^{r_{\min }+1} \rightarrow \mathcal{P}^{r_{\min }+1} \rightarrow \ldots
$$

of $R$-modules, in which the position in the complex is determined by the $\mathrm{U}(1)_{R}$-charge. This complex is bounded to the left, but unbounded to the right. The desired complex $\tilde{\mathcal{P}}$ of coherent sheaves on the projective hypersurface $X=\operatorname{Proj}(R)$ in $\mathbb{P}^{N-1}$ is obtained from $\mathcal{P}$ by sheafification. In particular, free $R$-modules $R[q]$ with $\mathrm{U}(1)$-representation specified by $q \in \mathbb{Z}$ which appear in $\mathcal{P}$ give rise to sheaves $\mathcal{O}_{X}(-q)$ on $X$ in $\tilde{\mathcal{P}}$. A priori this is a complex of coherent sheaves on $X$ which is unbounded to the right, but in fact it is quasi-isomorphic to a bounded complex [22]. In this way, one obtains the large volume interpretation of the GLSM B-brane $\hat{P}$. In the next subsection we will present a simple example of this construction.

\subsection{Example: tensor product branes}

As an example let us discuss how to transport tensor product B-branes from the Landau-Ginzburg to the large volume phase. These B-branes exist 
for any Landau-Ginzburg model with homogeneous superpotential. So let $W \in S:=\mathbb{C}\left[x_{1}, \ldots, x_{N}\right]$ be a homogeneous polynomial of degree $d$. Then $W$ can be written as

$$
W\left(x_{i}\right)=\sum_{j} x_{j} A_{j}\left(x_{i}\right)
$$

for some homogeneous polynomials $A_{j}$ of degree $d-1$. The $A_{j}$ are not unique in general, but the equivalence class of the tensor product $P=$ $\bigotimes_{j} P(j)$ of rank-one matrix factorizations

$$
P(j): P(j)_{1} \cong S \underset{p(j)_{0}=A_{j}}{\stackrel{p(j)_{1}=x_{j}}{\rightleftarrows}} S \cong P(j)_{0}
$$

of $x_{j} A_{j}$ is independent of the choice. Being a tensor product matrix factorization,

$$
P: P_{1} \underset{p_{0}}{\stackrel{p_{1}}{\rightleftarrows}} P_{0}
$$

has the following form:

$$
\begin{aligned}
P_{s} & =\bigoplus_{\substack{\left.s_{i}\right) \in \mathbb{Z}_{2}^{N} \\
s-\sum_{i} s_{i} \text { even }}} \bigotimes_{i=1}^{N} P(i)_{s_{i}}, \\
p_{s} & =\sum_{\substack{\left(s_{i}\right) \in \mathbb{Z}_{2}^{N} \\
s-\sum_{i} s_{i} \text { even }}} \sum_{j=1}^{N}(-1)^{\sum_{k=1}^{j-1} s_{k}} \operatorname{id}_{P(1)_{s_{1}}} \otimes \ldots \otimes p(j)_{s_{j}} \otimes \ldots \otimes \operatorname{id}_{P(N)_{s_{N}}} .
\end{aligned}
$$

Indeed, there is a slightly more elegant Koszul-type representation of this matrix factorization (cf. [40, Section 4.3]), which will be very useful later for the extraction of the large volume complex. In order to describe it, one introduces the vector space $V=S^{N}$ with basis $\left(e_{i}\right)_{i}$ and dual basis $\left(e_{i}^{*}\right)_{i}$. Then $P$ can be written as

$$
P: \Lambda^{\text {odd }} V \underset{\delta+\sigma}{\stackrel{\delta+\sigma}{\rightleftarrows}} \Lambda^{\text {even }} V
$$

with $^{9}$

$$
\delta=\imath_{\sum_{j} x_{j} e_{j}^{*}}, \quad \sigma=\left(\sum_{j} A_{j} e_{j}\right) \wedge \cdot
$$

\footnotetext{
${ }^{9}$ Note that $\delta \sigma+\sigma \delta=W$ id.
} 
As a side remark let us point out that the Koszul representation for tensor product matrix factorization appears very naturally in the discussion of Btype boundary conditions in Landau-Ginzburg models. There, one typically introduces additional boundary fermions $\psi_{i}$ and their duals $\psi_{i}^{*}$ in order to achieve B-type supersymmetry despite the presence of the boundary [41-43]. These fermions satisfy a Clifford algebra with relations $\left\{\pi_{i}, \pi_{j}^{*}\right\}=\delta_{i j}$, and their presence gives rise to the boundary contribution

$$
Q_{\mathrm{bdry}}=\sum_{j}\left(A_{j} \pi_{j}^{*}+x_{j} \pi_{j}\right)
$$

of the supercharge. In the Koszul representation, these fermions are realized by the operators

$$
\pi_{i}=e_{i} \wedge \cdot, \quad \pi_{i}^{*}=\imath_{e_{i}^{*}},
$$

with corresponding Fock space $\Lambda^{*} V$. The supercharge can be easily recognized to be the matrix factorization map $Q_{\mathrm{bdry}}=\delta+\sigma$ in this representation.

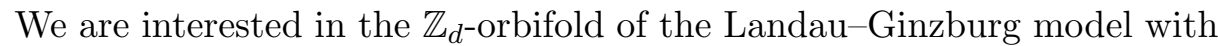
superpotential $W$, where $\mathbb{Z}_{d}$ acts on the $x_{i}$ by collective phase multiplications. The B-branes we would like to consider here are the ones associated to the matrix factorizations obtained from $P$ by means of the orbifold construction. Being invariant under $\mathbb{Z}_{d}$, the orbifold just introduces $\mathbb{Z}_{d}$-representations on the modules $P_{s}$. The result are the following

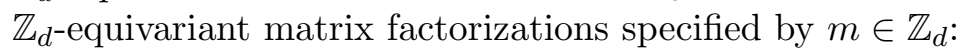

$$
\tilde{P}^{m}: \tilde{P}_{1}^{m} \underset{\tilde{P}_{0}^{m}}{\stackrel{\tilde{P}_{1}^{m}}{\rightleftarrows}} \tilde{P}_{0}^{m},
$$

with

$$
\tilde{P}_{s}^{m}=\bigoplus_{\substack{\left(s_{i}\right) \in \mathbb{Z}_{2}^{N} \\ s-\sum_{i} s_{i} \text { even }}}\left(\bigotimes_{i=1}^{N} P(i)_{s_{i}}\right)\left[m+\sum_{i} s_{i}\right]
$$

and the maps $\tilde{P}_{s}^{m}=p_{s}$. Here [.] denotes the $\mathbb{Z}_{d}$-representation on the respective modules. The Koszul-type representation can be made equivariant as well by setting the $\mathbb{Z}_{d}$-degree $\left[e_{i}\right]$ of the basis vectors $e_{i}$ of $V$ to be 1 . Then, $\tilde{P}^{m}$ can be written as

$$
\tilde{P}^{m}:\left(\Lambda^{\text {odd }} V\right)[m] \underset{\delta+\sigma}{\stackrel{\delta+\sigma}{\rightleftarrows}}\left(\Lambda^{\text {even }} V\right)[m]
$$

with maps $\delta$ and $\sigma$ as above. 
The $\mathrm{U}(1)_{R}$-representations can be specified by lifting $m$ to be an element

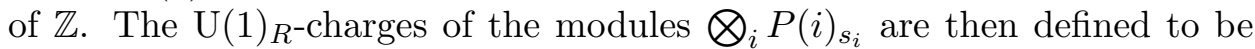
$\frac{2}{d}\left(m+\sum_{i}\left(s_{i}-d\right)\right)$. In the Koszul-representation this amounts to giving $R$ charge $-1+\frac{2}{d}$ to the basis vectors $e_{i}$ and shifting the overall $R$-charge of the $\Lambda^{s} V$ by $\frac{2 m}{d}$.

As discussed in Section 3 the Landau-Ginzburg orbifold can be obtained as a limit of a gauged linear $\sigma$-model with gauge group $\mathrm{U}(1)$, which in the LG-phase is broken down to $\mathbb{Z}_{d}$. Apart from the fields $x_{j}$ of charge 1 which are also present in the LG-model, there is an additional chiral field $\mathfrak{p}$ of charge $-d$ in the GLSM. The $R$-charge of the fields $x_{j}$ and $\mathfrak{p}$ are 0 and 2 , respectively, and the superpotential is given by $\hat{W}\left(x_{j}, \mathfrak{p}\right)=\mathfrak{p} W\left(x_{j}\right)$.

To transport B-branes from the LG-point to the large volume point of the Kähler moduli space, we follow [22] and first lift them to the GLSM. Thus, as discussed in Section 3.2 we need to construct $\mathrm{U}(1) \times \mathrm{U}(1)_{R^{-} \text {-equivariant }}$ matrix factorizations which at the Landau-Ginzburg point reduce to the

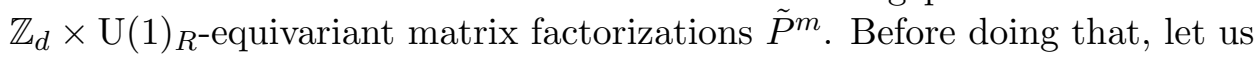
first discuss the lifts of the tensor factors

$$
\tilde{P}(j)^{m}: S[m+1] \underset{\tilde{P}(j)_{0}^{m}=A_{j}}{\stackrel{\tilde{P}(j)_{1}^{m}=x_{j}}{\rightleftarrows}} S[m]
$$

to the GLSM. Each $\tilde{P}(j)^{m}$ has two types of lifts:

$$
\hat{P}(j)^{[\alpha, a, r]}: \hat{S}[a+1-d \alpha, r-1+2 \alpha] \underset{\hat{p}(j)_{0}^{[\alpha, a, r]}=\mathfrak{p}^{1-\alpha} A_{j}}{\stackrel{\left.\hat{p}(j)\right|_{1} ^{[\alpha, a, r]}=p^{\alpha} x_{j}}{\rightleftarrows}} \hat{S}[a, r]
$$

$m=a \bmod d, \alpha \in\{0,1\}$ which differ in which of the maps $\tilde{P}_{s}^{m}$ is multiplied by $\mathfrak{p}$. Here $\hat{S}=\mathbb{C}\left[x_{i}, \mathfrak{p}\right]$ and $[\cdot, \cdot]$ denotes the $\mathrm{U}(1) \times \mathrm{U}(1)_{R}$-representations. Moreover, the lifted $R$-charge is given by $r=2 \frac{m-a}{d}$.

For the lift to the GLSM and the transport of the B-branes corresponding to $\tilde{P}^{m}$ to the large volume phase, we restrict the discussion to the "CalabiYau"-case, so from now on $d=N$. It is not difficult to see that in that case, there is only one lift of each $\tilde{P}^{m}$ which is compatible with a given charge window $\mathcal{N}_{\eta}=\{1-d+\eta, \ldots, \eta\}$. This lift is given in the following way. First, choose $a=m-d k \in \mathcal{N}_{\eta}$ for some $k \in \mathbb{Z}$ and give $\mathrm{U}(1) \times \mathrm{U}(1)_{R^{-}}$ representation $[a, r=2 k]$ to $\bigotimes_{j} P(j)_{0}$. Then, in the tensor product maps $\tilde{P}_{s}^{m}=p_{s}$ written in (3.6) one replaces the $p(j)_{s}$ by $\hat{p}(j)_{s}^{[0, \cdot, \cdot]}$ everywhere 
except in the $(n=1+\eta-a)$ th step

$$
\bigoplus \underset{\substack{\left(s_{1}, \ldots, s_{N}\right) \\ \sum_{i} s_{i}=n}}{ } \bigotimes_{j} P(j)_{s_{j}} \rightleftarrows \underset{\substack{\left(s_{1}, \ldots, s_{N}\right) \\ \sum_{i} s_{i}=n-1}}{\bigoplus} \bigotimes_{j} P(j)_{s_{j}}
$$

where one replaces them by $\hat{p}(j)_{s}^{[1, \cdot, \cdot]}$. Noting that

$$
\operatorname{source}\left(\hat{p}(j)_{1}^{[\alpha, a, r]}\right)=\operatorname{target}\left(\hat{p}(j)_{1}^{[\beta, a+1-d \alpha, r-1+2 \alpha]}\right)
$$

one arrives at the following matrix factorization:

$$
\hat{P}^{[n, a, r]}: \hat{P}_{1}^{[n, a, r]} \underset{\hat{p}_{0}^{[n, a, r]}}{\stackrel{\hat{p}_{1}^{[n, a, r]}}{\rightleftarrows}} \hat{P}_{0}^{[n, a, r]},
$$

with

$$
\begin{aligned}
\hat{P}_{s}^{[n, a, r]}= & \bigoplus_{\substack{\left(s_{i}\right) \in \mathbb{Z}_{2}^{N} \\
s-\sum_{i} s_{i} \text { even }}}\left(\bigotimes_{i=1}^{N} P(i)_{s_{i}}\right) \\
& {\left[a+\sum_{i} s_{i}-d \Theta\left(\sum_{i} s_{i}-n\right), r-\sum_{i} s_{i}+2 \Theta\left(\sum_{i} s_{i}-n\right)\right] }
\end{aligned}
$$

and

$$
\begin{aligned}
\hat{p}_{s}^{[n, a, r]}= & \sum_{\substack{\left(s_{i}\right) \in \mathbb{Z}_{2}^{N} \\
s-\sum_{i} s_{i} \text { even }}} \sum_{j=1}^{N}(-1)^{\sum_{k=1}^{j-1} s_{k}} \operatorname{id}_{P(1)_{s_{1}}} \otimes \ldots \\
& \ldots \otimes \hat{p}(j)_{s_{j}}^{[\delta(S(j, s)-n), a+S(j, s)-d \Theta(S(j, s)-n), r-S(j, s)+2 \Theta(S(j, s)-n)]} \otimes \ldots \\
& \ldots \otimes \operatorname{id}_{P(N)_{s_{N}}},
\end{aligned}
$$

where $S(j, s)=\sum_{k \neq j} s_{k}$.

In the Koszul-type representation this can be written as follows. First, set $\hat{V}:=\hat{S}^{N}$, and give degree $[1,-1]$ to its basis vectors $e_{i}$. Then $\hat{P}^{[n, a, r]}$ 
can be written as

$$
\begin{aligned}
\hat{P}^{[n, a, r]}: & \bigoplus_{\substack{s \text { odd } \\
\text { ing }}}\left(\Lambda^{s} \hat{V}\right)[a-d \Theta(s-n), r+2 \Theta(s-n)] \\
& \stackrel{\hat{\delta}+\hat{\sigma}}{\rightleftarrows} \bigoplus_{\hat{\delta}+\hat{\sigma}}\left(\Lambda^{s} \hat{V}\right)[a-d \Theta(s-n), r+2 \Theta(s-n)],
\end{aligned}
$$

where

$$
\left.\hat{\delta}\right|_{\Lambda^{s} \hat{V}}=\left\{\left.\begin{array}{ll}
\delta, & \text { for } s \neq n, \\
\mathfrak{p} \delta, & \text { for } s=n,
\end{array} \quad \hat{\sigma}\right|_{\Lambda^{s} \hat{V}}= \begin{cases}\mathfrak{p} \sigma, & \text { for } s \neq n-1 \\
\sigma, & \text { for } s=n-1\end{cases}\right.
$$

Let us transport the B-branes corresponding to $P^{m}$ from the LandauGinzburg to the large volume phase through the phase boundary component $N_{0}$. The lift of $P^{m}$ compatible with the corresponding charge window $\mathcal{N}_{0}=\{1-d, \ldots, 0\}$ is given by $\hat{P}^{[1-a, a, r]}$, where $a=m-d k \in \mathcal{N}_{0}$ for some $k \in \mathbb{Z}$, and $r=2 k$. We now follow [22] to construct the B-brane obtained by transporting the LG-brane associated to $\tilde{P}^{m}$ to the large volume phase along a path which traverses the phase boundary in the segment $N_{0}$. As described in Section 3.3 we first have to tensor the matrix factorization $\hat{P}^{[1-a, a, r]}$ by $\hat{S} /(W)$. Then we regard it as a complex over $R:=\hat{S} /(W, \mathfrak{p})$ and "unfold" it with respect to $\mathrm{U}(1)_{R}$-charge. The sheafification of the resulting complex represents the large volume B-brane. For the case at hand, we use the Koszul-type representation of $\hat{P}^{[1-a, a, r]}$ and introduce $V_{R}:=\hat{V} \otimes R$. Then it is not difficult to see that the resulting complex indeed has the form

$$
\operatorname{Cone}\left(\mathcal{K}^{-a} \stackrel{\sigma}{\rightarrow} \mathcal{C}\right)\{-r\}
$$

where $\{\cdot\}$ denotes the shift of complexes and $\mathcal{K}^{-a}$ is the complex

$$
\mathcal{K}^{-a}: \Lambda^{-a} V_{R}[a] \stackrel{\delta}{\rightarrow} \Lambda^{-a-1} V_{R}[a] \stackrel{\delta}{\rightarrow} \ldots \stackrel{\delta}{\rightarrow} \Lambda^{0} V_{R}[a] \rightarrow 0
$$

starting at position $a$. Moreover, $\mathcal{C}$ is an infinite complex which itself can be written as successive cone:

$$
\mathcal{C}=\operatorname{Cone}\left(\mathcal{K}^{d}\{-2\} \otimes R[a] \stackrel{\sigma}{\rightarrow} \mathcal{C}\{-2\} \otimes R[-d]\right)
$$

Now $\mathcal{K}^{t}$ can be identified as the dual of the Koszul complex of $\left(x_{i}\right)$ truncated at the $-(t+1)$ st position. Its sheafification $\tilde{\mathcal{K}}^{t}$ is exact except at the $-t$ th position with cohomology $\tilde{M}_{t}$. It is a well-known fact (cf. e.g., [44, Chapter 5B] - for more details on the Koszul complex see, e.g., [45]) that the 
Table 1: The first column shows a collection of tensor product matrix factorizations describing B-branes in the Landau-Ginzburg phase. Their lifts to the gauged linear $\sigma$-model compatible with charge window $\mathcal{N}_{0}$ are listed in the second column. The third column contains the large volume B-branes obtained by transporting the LG-branes through the phase boundary component $N_{0}$ into the large volume phase. Here underlining marks position zero in complexes.

\begin{tabular}{lcc}
\hline LG brane & GLSM brane & LV brane \\
\hline$\vdots$ & $\vdots$ & $\vdots$ \\
$\tilde{P}^{0}$ & $\hat{P}^{[1,0,0]}$ & $\left.\Omega_{\mathbb{P}^{N-1}}^{0}\right|_{X}(0) \cong \underline{\mathcal{O}_{X}}$ \\
$\tilde{P}^{-1}$ & $\hat{P}^{[2,-1,0]}$ & $\left.\Omega_{\mathbb{P}^{N-1}}^{1}(1)\right|_{X}\{1\} \cong \mathcal{O}_{X}^{\oplus d} \rightarrow \underline{\mathcal{O}_{X}(1)}$ \\
$\tilde{P}^{-2}$ & $\hat{P}^{[3,-2,0]}$ & $\left.\Omega_{\mathbb{P}^{N-1}}^{2}(2)\right|_{X}\{2\} \cong \mathcal{O}_{X}^{\oplus\left(\begin{array}{c}d \\
2\end{array}\right)} \rightarrow \mathcal{O}_{X}(1)^{\oplus d} \rightarrow \underline{\mathcal{O}_{X}(2)}$ \\
$\vdots$ & $\vdots$ & $\vdots$ \\
$\tilde{P}^{-(d-1)}$ & $\hat{P}^{[d, 1-d, 0]}$ & $\left.\mathcal{O}_{X}^{\oplus\left({ }_{d-1}^{d}\right)}\right) \rightarrow \cdots \rightarrow \mathcal{O}_{X}(d-2)^{\oplus\left(\begin{array}{l}d \\
1\end{array}\right) \rightarrow \mathcal{O}_{X}(d-1)}$ \\
$\tilde{P}^{-d}$ & $\hat{P}^{[1,0,-2]}$ & $\left.\Omega_{\mathbb{P}^{N-1}}^{d-1}(d-1)\right|_{X}(0)\{2\} \cong \mathcal{O}_{X}\{2\}$ \\
$\vdots$ & $\vdots$ & $\vdots$ \\
\hline
\end{tabular}

sheafification of $M_{t}$ is given by

$$
\left.\tilde{M}_{t} \cong \Omega_{\mathbb{P}^{N-1}}^{t}(t)\right|_{X},
$$

where $\left.\Omega_{\mathbb{P}^{N-1}}^{t}(t)\right|_{X}$ is the vector bundle $\Lambda^{t} T^{*} \mathbb{P}^{N-1} \otimes \mathcal{O}(t)$ on the projective space $\mathbb{P}^{N-1}$ restricted to the hypersurface $X$. It is zero for $t \geq N$. Thus, $\tilde{\mathcal{K}}^{t}$ is quasi-isomorphic to

$$
\left.\tilde{\mathcal{K}}^{t} \cong \Omega_{\mathbb{P}^{N-1}}^{t}(t)\right|_{X}\{t\},
$$

whereas $\tilde{\mathcal{K}}^{d}$ is quasi-isomorphic to the trivial complex. The latter implies that $\mathcal{C}$ is quasi-isomorphic to the trivial complex as well, and therefore, as summarized in table 1 , we obtain the large volume B-brane

$$
\left.\operatorname{Cone}\left(\tilde{\mathcal{K}}^{-a} \stackrel{\tilde{\sigma}}{\rightarrow} \tilde{\mathcal{C}}\right)\{-2 k\} \cong \tilde{\mathcal{K}}^{-a}\{-2 k\} \cong \Omega_{\mathbb{P}^{N-1}}^{-a}(-a)\right|_{X}\{-a-2 k\},
$$

by transporting the Landau-Ginzburg B-brane associated to the matrix factorization $\tilde{P}^{m},\left(m=a+d k, a \in \mathcal{N}_{0}, k \in \mathbb{Z}\right)$ into the large volume phase along a path intersecting the phase boundary in $N_{0}$. This is indeed the expected result [46]. 


\subsection{Defects}

As discussed in Section 2, at the Landau-Ginzburg point, defects can be described by matrix factorizations $D$ of $W_{1}\left(x_{1}, \ldots, x_{N}\right)-W_{2}\left(y_{1}, \ldots, y_{M}\right)$. As such they can be transported to the large volume point in the same way as B-type D-branes. Namely, they can be lifted to defects between two GLSMs, i.e., to matrix factorizations of $\mathfrak{p} W_{1}\left(x_{1}, \ldots, x_{N}\right)-\mathfrak{q} W\left(y_{1}, \ldots, y_{M}\right)$. This provides a uniform description of B-type defects on the Kähler moduli space of the product of the two GLSMs. The latter is the product of the two Kähler moduli spaces. In particular, one can transport B-type defects through the product $N \times N^{\prime}$ of phase boundary components of the respective models to the product of their large volume points. In this way, one obtains a complex $\mathcal{D}$ of coherent sheaves on the product $X \times Y$ of projective hypersurfaces $X=\left\{W_{1}=0\right\} \subset \mathbb{P}^{N-1}, Y=\left\{W_{2}=0\right\} \subset \mathbb{P}^{M-1}$ which represents the defect at large volume. We will now argue that this defect acts on the category $D^{b}(Y)$ as Fourier-Mukai transformation with kernel given by $\mathcal{D} \otimes\left(\mathcal{O}_{X} \otimes\right.$ $\left.\mathcal{O}_{Y}(d)\{-1\}\right)$.

Let us recall some basic facts about Fourier-Mukai transformations (for more details see, e.g., $[47,48])$. To every object $\mathcal{R} \in D^{b}(X \times Y)$ one can associate a functor

$$
\Phi^{\mathcal{R}}: D^{b}(Y) \rightarrow D^{b}(X), \quad \Phi^{\mathcal{R}}(\cdot)=\mathbf{R} \pi_{1 *}\left(\mathcal{R} \stackrel{\mathbf{L}}{\otimes} \mathbf{L} \pi_{2}^{*}(\cdot)\right),
$$

where $\pi_{i}$ denote the projections $X \stackrel{\pi_{1}}{\leftarrow} X \times Y \stackrel{\pi_{2}}{\rightarrow} Y$ on the factors, and "L" and "R" indicate that the tensor product, the pullback and the pushforward are left- and right-derived, respectively. ${ }^{10}$ In particular, for $\mathcal{F} \in D^{b}(X)$, $\mathcal{G} \in D^{b}(Y)$ this functor satisfies the following formula:

$$
\operatorname{Hom}\left(\mathcal{F} \otimes \mathcal{G}^{\vee}, \mathcal{R}\right) \cong \operatorname{Hom}\left(\mathcal{F}, \Phi^{\mathcal{R}}(\mathcal{G})\right)
$$

Here we used the abbreviation $\mathcal{F} \otimes \mathcal{G}=\pi_{1}^{*}(\mathcal{F}) \otimes \pi_{2}^{*}(\mathcal{G})$.

Now, let us turn back to the Landau-Ginzburg point. Let $Q$ and $P$ be matrix factorizations of $W_{1}\left(x_{i}\right)$ and $W_{2}\left(y_{i}\right)$, respectively, representing Btype D-branes in the corresponding Landau-Ginzburg models, and let $D$ be a matrix factorization of $W_{1}\left(x_{i}\right)-W_{2}\left(y_{i}\right)$ describing a defect between these

\footnotetext{
${ }^{10}$ For an introduction to derived categories and derived functors see, e.g., [49]. In the following for ease of notation we do not explicitly denote in our formulae the left- or right-derived property of a functor.
} 
models. Then the following "folding formula" holds: ${ }^{11}$

$$
\mathcal{H}(Q, D * P) \cong \mathcal{H}\left(Q \otimes P^{*}, D\right),
$$

where the dual $P^{*}$ of a matrix factorization $P$ is defined by

$$
\left(P_{1} \underset{p_{0}}{\stackrel{p_{1}}{\rightleftarrows}} P_{0}\right)^{*}=\left(P_{1}^{*} \underset{-p_{1}^{*}}{\stackrel{p_{0}^{*}}{\rightleftarrows}} P_{0}^{*}\right) .
$$

To transport these matrix factorizations to large volume, we fix components $N$ and $N^{\prime}$ of the phase boundary in the Kähler moduli space of the two models. Let us denote by $\mathrm{LV}_{W}$ the parallel transport from the LandauGinzburg point to large volume through the component $W$ of the phase boundary. Then define the complexes

$$
\begin{aligned}
& \mathcal{Q}:=\operatorname{LV}_{N}(Q), \quad \mathcal{P}:=\operatorname{LV}_{N^{\prime}}(P), \quad \mathcal{P}^{*}:=\operatorname{LV}_{-N^{\prime}}\left(P^{*}\right), \quad \mathcal{F}:=\operatorname{LV}_{N}(D * P), \\
& \mathcal{T}:=\operatorname{LV}_{N \times\left(-N^{\prime}\right)}\left(Q \otimes P^{*}\right), \quad \mathcal{D}:=\operatorname{LV}_{N \times\left(-N^{\prime}\right)}(D) .
\end{aligned}
$$

Note that $-N^{\prime}$ is the component of the phase boundary dual to $N^{\prime}$, i.e., the corresponding charge windows satisfy $\mathcal{N}^{\prime}=-\mathcal{N}$.

Since transport to large volume is an equivalence of categories (3.31) implies

$$
\operatorname{Hom}(\mathcal{Q}, \mathcal{F}) \cong \operatorname{Hom}(\mathcal{T}, \mathcal{D})
$$

But it is not difficult to see that indeed

$$
\mathcal{T} \cong \mathcal{Q} \otimes \mathcal{P}^{*} \cong Q \otimes \mathcal{P}^{\vee} \otimes \mathcal{O}_{Y}(-d)\{1\}
$$

where $\mathcal{P}^{\vee}$ denotes the dual complex of $\mathcal{P}$. In the second step we used the relation

$$
\mathcal{P}^{*} \cong \mathcal{P}^{\vee} \otimes \mathcal{O}_{Y}(-d)\{1\}
$$

between duality of matrix factorization and duality of large volume complexes shown in Appendix A. Therefore, using (3.30) we arrive at

$$
\begin{aligned}
\operatorname{Hom}(\mathcal{Q}, \mathcal{F}) & \cong \operatorname{Hom}\left(\mathcal{Q} \otimes\left(\mathcal{P} \otimes \mathcal{O}_{Y}(d)\{-1\}\right)^{\vee}, \mathcal{D}\right) \\
& \cong \operatorname{Hom}\left(\mathcal{Q}, \Phi^{\mathcal{D} \otimes\left(\mathcal{O}_{X} \otimes \mathcal{O}_{Y}(d)\{-1\}\right)} \mathcal{P}\right) .
\end{aligned}
$$

\footnotetext{
${ }^{11}$ This is obvious from the folding trick, in which defects between two theories are regarded as boundary conditions in the tensor product of the first theory and the worldsheet parity dual of the second one.
} 
From this we deduce that fusion with the defect $D$ transported from the Landau-Ginzburg point to large volume through the component $N \times\left(-N^{\prime}\right)$ is realized by the Fourier-Mukai transform $\Phi^{\mathcal{R}}$ with kernel

$$
\mathcal{R} \cong \mathcal{D} \otimes\left(\mathcal{O}_{X} \otimes \mathcal{O}_{Y}(d)\{-1\}\right)
$$

\section{Landau-Ginzburg monodromy}

The monodromies which are most easily described in the Landau-Ginzburg framework are those around the Landau-Ginzburg points themselves. These are orbifold points in Kähler moduli space, at which certain dualities become self-dualities. As a consequence, the symmetry groups of the theory at these points are enhanced by "quantum symmetries," which in turn realize the monodromies around these points.

The models we are interested in here are one-parameter models which can be realized as gauged linear $\sigma$-models with $\mathrm{U}(1)$ gauge group, chiral superfields $\left(\mathfrak{p}, x_{1}, \ldots, x_{N}\right)$ of charge $(-d, 1, \ldots, 1)$ and superpotential $\mathfrak{p} W\left(x_{1}, \ldots, x_{N}\right)$, where $W \in \mathbb{C}\left[x_{1}, \ldots, x_{N}\right]$ is homogeneous of degree $d$. At the Landau-Ginzburg point, the field $\mathfrak{p}$ receives a non-vanishing vacuum expectation value, and the model degenerates to a Landau-Ginzburg orbifold with chiral superfields $x_{i}$, superpotential $W$ and orbifold group $\mathbb{Z}_{d}$ to which the gauge group is broken.

The action of the quantum symmetries in these orbifolds are well known. They act by phase multiplications in the twisted sectors. On the level of D-branes, this action is given by a shift in the representations of the orbifold group $\mathbb{Z}_{d}$ and the $R$-symmetry group $\mathrm{U}(1)_{R} \cdot{ }^{12}$

Using the results of [11] one can easily construct defect matrix factorizations realising precisely this shift.

\subsection{Landau-Ginzburg phase}

In this section we will construct the graded matrix factorizations representing the defects associated to Landau-Ginzburg monodromies. Let $W \in$

\footnotetext{
${ }^{12}$ This is rather obvious from the gauged linear $\sigma$-model perspective [22], where boundary actions only depend on the $\theta$-angle through the combination $\theta+2 \pi q$. Thus going around the Landau-Ginzburg point $\theta \mapsto \theta+2 \pi$ shifts the U(1)-representations $q$ of D-branes by $q \mapsto q-1$.
} 
$\mathbb{C}\left[x_{1}, \ldots, x_{N}\right]$ be a homogeneous polynomial of degree $d$. We assume that the orbifold group $\Gamma=\mathbb{Z}_{d}$ acts on the $x_{i}$ by a collective phase multiplication.

In the unorbifolded Landau-Ginzburg model, the identity defect is represented by the tensor product matrix factorization (see, e.g., [11])

$$
I=\bigotimes_{i=1}^{N} I(i)
$$

of the rank-one matrix factorizations

$$
I(i): I(i)_{1}=S \underset{\imath(i)_{0}}{\stackrel{\imath(i)_{1}}{\rightleftarrows}} S=I(i)_{0}, \imath(i)_{1}=\left(x_{i}-y_{i}\right), \imath(i)_{0}=A_{i}\left(x_{j}, y_{j}\right)
$$

Here $S=\mathbb{C}\left[x_{j}, y_{j}\right]$ and $A_{i}$ are homogeneous polynomials of degree $d-1$ such that ${ }^{13} W\left(x_{j}\right)-W\left(y_{j}\right)=\sum_{i=1}^{N}\left(x_{i}-y_{i}\right) A_{i}\left(x_{j}, y_{j}\right)$.

The graded matrix factorizations corresponding to the Landau-Ginzburg monodromies in the $\Gamma$-orbifold can be obtained from $I$ by means of the orbifold construction. For the case of Landau-Ginzburg models with one chiral field, i.e., $N=1$ this has been discussed in [12]. Let us briefly review this construction for the rank-one factorizations (4.2). Since the defects separate two orbifold theories, they have to be orbifolded by the product of the orbifold groups on either side. In this case the group is $\hat{\Gamma}=\Gamma \times \Gamma=$ $\mathbb{Z}_{d} \times \mathbb{Z}_{d}$, where the first factor acts on the variables $x_{j}$ and the second one on the variables $y_{j}$ only. As usual, the orbifold procedure requires to choose on the given unorbifolded matrix factorization a representation of the subgroup $\hat{\Gamma}_{s} \subset \hat{\Gamma}$ which stabilizes it. Then one sums over its orbit under $\hat{\Gamma} / \hat{\Gamma}_{s}$. The result is a $\hat{\Gamma}$-equivariant matrix factorization.

The matrix factorizations $I(i)$ are invariant under the diagonal subgroup $\Gamma_{s}=\Gamma_{\text {diag }} \subset \Gamma \times \Gamma$, because the maps $\imath(i)_{s}$ are homogeneous polynomials. Therefore one has to choose a representation of $\Gamma \cong \Gamma_{s}$ on it, which we will denote by $m \in \mathbb{Z}_{d}$. The next step is to sum up the $\Gamma \times \Gamma / \Gamma_{\text {diag }} \cong \Gamma$ orbit. The result is a direct sum of $d$ rank-one factorizations. By construction the action of $\Gamma \times \Gamma$ is not diagonal in the sum basis, but one can diagonalize it. In the corresponding basis the resulting $\Gamma \times \Gamma$-equivariant matrix factorizations

\footnotetext{
${ }^{13}$ Although the $A_{i}$ are not determined uniquely by this equation, the equivalence class of $I$ is independent of the choices of the $A_{i}$.
} 
$\tilde{I}(i)^{m}$ are given by

$$
\tilde{I}(i)^{m}: \tilde{I}(i)_{1}^{m}=V^{m+1} \underset{\tilde{\imath}(i)_{0}^{m}}{\stackrel{\tilde{\imath}(i)_{1}^{m}}{\rightleftarrows}} V^{m}=\tilde{I}(i)_{0}^{m},
$$

where

$$
\begin{aligned}
V^{m} & =\bigoplus_{\nu=0}^{d-1} S[m+\nu,-\nu], \\
\tilde{\imath}(i)_{1}^{m} & =\left(\begin{array}{cccc}
x_{i} & & & -y_{i} \\
-y_{i} & \ddots & & \\
& \ddots & \ddots & \\
& & -y_{i} & x_{i}
\end{array}\right), \quad\left(\tilde{\imath}(i)_{0}^{m}\right)_{\mu \nu}=A_{i}^{\mu-\nu}\left(x_{j}, y_{j}\right) .
\end{aligned}
$$

Here $[\cdot, \cdot \cdot]$ specifies the $\mathbb{Z}_{d} \times \mathbb{Z}_{d}$-representation on this module, and $A_{i}^{l}\left(x_{j}, y_{j}\right)$ are the degree $[d-1-l, l]$-parts of the polynomials $A_{i}\left(x_{j}, y_{j}\right)$. To write this in a more compact form, denote by $\left(f_{\mu}^{m}\right)_{\mu}$ the basis of $V^{m}$ with degrees $\left[f_{\mu}^{m}\right]=[m+\mu,-\mu]$, and define the map $\tau: V^{m} \rightarrow V^{m}, f_{\mu}^{m} \mapsto f_{\mu+1 \bmod d}^{m}$. Then

$$
\tilde{\imath}(i)_{1}^{m}=x_{i} \mathrm{id}-y_{i} \tau, \quad \tilde{\imath}(i)_{0}^{m}=\sum_{l=0}^{d-1} A_{i}^{l}\left(x_{j}, y_{j}\right) \tau^{l} .
$$

For a more detailed discussion see [12].

Indeed, this construction can easily be generalized to the matrix factorization (4.1) corresponding to the identity defect. As tensor product the latter has the following form:

$$
I: I_{1} \underset{\imath_{0}}{\stackrel{\imath_{1}}{\rightleftarrows}} I_{0}
$$

where

$$
I_{s}=\bigoplus_{\substack{\left(s_{i}\right) \in \mathbb{Z}_{2}^{N} \\ s-\sum_{i} s_{i} \text { even }}} \bigotimes_{i=1}^{N} I(i)_{s_{i}}
$$

and

$$
\imath_{s}=\sum_{\substack{\left(s_{i}\right) \in \mathbb{Z}_{2}^{N} \\ s-\sum_{i} s_{i} \text { even }}} \sum_{j=1}^{N}(-1)^{\sum_{k=1}^{j-1} s_{k}} \operatorname{id}_{I(1)_{s_{1}}} \otimes \ldots \otimes \imath(j)_{s_{j}} \otimes \ldots \otimes \operatorname{id}_{I(N)_{s_{N}}} .
$$


As in the case of tensor product B-branes discussed in Section 3.4, a Koszultype representation of this matrix factorization is useful. Let $V:=S^{N}$ with basis $\left(e_{i}\right)_{i}$ and denote the dual basis by $\left(e_{i}^{*}\right)_{i}$. Then the identity matrix factorization (4.1) can be represented by

$$
I: \Lambda^{\text {odd }} V \underset{\delta+\sigma}{\stackrel{\delta+\sigma}{\rightleftarrows}} \Lambda^{\text {even }} V
$$

with $^{14}$

$$
\delta=\imath_{\sum_{i}\left(x_{i}-y_{i}\right) e_{i}^{*}}, \quad \sigma=\left(\sum_{i} A_{i} e_{i}\right) \wedge \cdot
$$

Applying the orbifold construction to this matrix factorization yields the equivariant matrix factorizations

$$
\tilde{I}^{m}: \tilde{I}_{1}^{m} \underset{\tilde{i}_{0}^{m}}{\stackrel{\tilde{\imath}_{1}^{m}}{\rightleftarrows}} \tilde{I}_{0}^{m}
$$

with

and

$$
\tilde{I}_{s}^{m}=\bigoplus_{\substack{\left(s_{i}\right) \in \mathbb{Z}_{2}^{N} \\ s-\sum_{i} s_{i} \text { even }}} \bigotimes_{i=1}^{N} I(i)_{s_{i}} \otimes V^{m+\sum_{i} s_{i}}
$$

$$
\begin{aligned}
\tilde{\imath}_{s}^{m}= & \sum_{\substack{\left(s_{i}\right) \in \mathbb{Z}_{2}^{N} \\
s-\sum_{i} s_{i} \text { even }}} \sum_{j=1}^{N}(-1)^{\sum_{k=1}^{j-1} s_{k}} \operatorname{id}_{I(1)_{s_{1}}} \otimes \ldots \otimes \tilde{\imath}(j)_{s_{j}}^{m+\sum_{k \neq j} s_{k}} \otimes \ldots \\
& \ldots \otimes \operatorname{id}_{I(N)_{s_{N}}} .
\end{aligned}
$$

To describe the result of the orbifold construction in the Koszulrepresentation (4.9), we set the $\mathbb{Z}_{d} \times \mathbb{Z}_{d}$-degree of the basis vectors $e_{i}$ of $V$ to $[1,0]$. Then the orbifolds of the identity matrix factorization can be written as

$$
\tilde{I}^{m}: \Lambda^{\text {odd }} V \otimes V^{m} \underset{\tilde{\delta}+\tilde{\sigma}}{\stackrel{\tilde{\delta}+\tilde{\sigma}}{\rightleftarrows}} \Lambda^{\text {even }} V \otimes V^{m}
$$

with

$$
\tilde{\delta}=\imath_{\sum_{i} x_{i} e_{i}^{*}} \otimes \mathrm{id}-\imath_{\sum_{i} y_{i} e_{i}^{*}} \otimes \tau, \quad \tilde{\sigma}=\sum_{i, l} A_{i}^{l}\left(e_{i} \wedge \cdot\right) \otimes \tau^{l} .
$$

\footnotetext{
${ }^{14} \mathrm{As}$ in the case of the tensor product D-branes discussed in Section $3.4 \delta \sigma+\sigma \delta=$ $\left(W\left(x_{j}\right)-W\left(y_{j}\right)\right)$ id.
} 
The $\mathrm{U}(1)_{R}$-representations on the matrix factorizations $\tilde{I}^{m}$ are specified as follows. Similarly to the case of tensor product D-branes discussed in Section $3.4, m$ is lifted to an integer, and the $R$-charge of vectors in $V^{m+\sum_{i} s_{i}}$ in (4.12) is defined to be $\frac{2}{d}\left(m+\sum_{i}\left(s_{i}-d\right)\right)$. In the Koszul-representation (4.14) one also sets the $R$-charge of vectors of $V^{m}$ to $\frac{2 m}{d}$, and the ones of the basis vectors $e_{i}$ of $V$ to $-1+\frac{2}{d}$.

\subsection{Lift to the GLSM}

Next, we will lift the matrix factorizations corresponding to LandauGinzburg monodromies which have been constructed in Section 4.1 to the gauged linear $\sigma$-model. Apart from the chiral fields $x_{i}$ which were also present in the Landau-Ginzburg model, this model contains an additional field $\mathfrak{p}$, and the gauge group which is broken to $\Gamma=\mathbb{Z}_{d}$ in the LandauGinzburg phase is $\mathrm{U}(1)$. The $x_{i}$ have charge 1 with respect to it, and the field $\mathfrak{p}$ charge $-d$. The model also exhibits a $\mathrm{U}(1)_{R}$, with charges 0 and 2 of the fields $x_{i}$ and $\mathfrak{p}$, respectively. The superpotential of the model is given by $\hat{W}\left(\mathfrak{p}, x_{j}\right)=\mathfrak{p} W\left(x_{j}\right)$. To lift a $\mathbb{Z}_{d} \times \mathrm{U}(1)_{R}$-equivariant matrix factorization $P$ of $W$ from the Landau-Ginzburg to the gauged linear $\sigma$ model means to construct $\mathrm{U}(1) \times \mathrm{U}(1)_{R}$-equivariant matrix factorizations of $\hat{W}$ which in the Landau-Ginzburg phase reduce to $P$. As alluded to in Section 3.2 there are in general several lifts of a given matrix factorization $P$. We are going to lift the defect matrix factorizations $\tilde{I}^{m}$ constructed

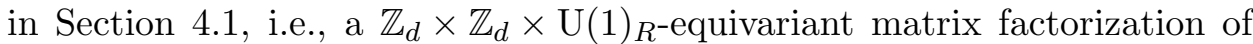
$W\left(x_{j}\right)-W\left(y_{j}\right)$ to $\mathrm{U}(1) \times \mathrm{U}(1) \times \mathrm{U}(1)_{R}$-equivariant matrix factorizations of $\hat{W}\left(\mathfrak{p}, x_{j}\right)-\hat{W}\left(\mathfrak{q}, y_{j}\right)$. In order to do so, we will again start by lifting the orbifold factorizations $\tilde{I}(i)^{m}$ of the tensor factors of the identity defect in the unorbifolded Landau-Ginzburg models.

It is not difficult to see how to promote the factorizations $\tilde{I}(i)^{m}$ to the gauged linear $\sigma$-model. To be able to lift the $\mathbb{Z}_{d} \times \mathbb{Z}_{d}$-representations to $\mathrm{U}(1) \times \mathrm{U}(1)$-representations, one needs to replace one of the entries $x_{i}$ of $\tilde{\imath}(i)_{1}^{m}$ by $\mathfrak{p} x_{i}$, and one of the entries $y_{i}$ by $\mathfrak{q} y_{i}$. At the same time, one has to introduce monomial factors in $\mathfrak{p}$ and $\mathfrak{q}$ in the entries of $\tilde{\imath}(i)_{0}^{m}$ accordingly. Once the factors of $\mathfrak{p}$ and $\mathfrak{q}$ have been placed in $\tilde{\imath}(i)_{1}^{m}$ the distribution of factors in $\tilde{\imath}(i)_{0}^{m}$ is fixed. The lifts of the matrix factorizations $\tilde{I}(i)^{m}$ are given by

$$
\hat{I}(i)^{[n, a, b, r]}: V^{[n+1, a+1, b, r-1]} \underset{\tilde{\imath}(i)_{0}^{[n, a, b, r]}}{\stackrel{\hat{\imath}(i){ }_{1}^{[n, a, b, r]}}{\rightleftarrows}} V^{[n, a, b, r]},
$$


with

$$
V^{[n, a, b, r]}=\bigoplus_{\nu=0}^{d-1} \hat{S}[a+\nu-d \Theta(\nu-d+n), b-\nu, r+2 \Theta(\nu-d+n)]
$$

and

$$
\begin{aligned}
\hat{\imath}(i)_{1}^{[n, a, b, r]} & =\left(\begin{array}{ccccc}
x_{i} & & & & -\mathfrak{q} y_{i} \\
-y_{i} & \ddots & & & \\
& \ddots & \mathfrak{p} x_{i} & & \\
& & \ddots & \ddots & \\
& & & -y_{i} & x_{i}
\end{array}\right), \\
\left(\hat{\imath}(i)_{0}^{[n, a, b, r]}\right)_{\mu \nu} & =Z^{\mu, \nu}(\mathfrak{p}, \mathfrak{q}) A_{i}^{\mu-\nu}\left(x_{j}, y_{j}\right) .
\end{aligned}
$$

Here $\quad \hat{S}=\mathbb{C}\left[x_{j}, y_{j}, \mathfrak{p}, \mathfrak{q}\right], \quad[\cdot, \cdot, \cdot] \quad$ specifies the $\mathrm{U}(1) \times \mathrm{U}(1) \times \mathrm{U}(1)_{R^{-}}$ representation and the entry $\mathfrak{p} x_{i}$ in $\hat{\imath}(i)_{1}^{[n, a, b, r]}$ is at the $(d-n)$ th position. The matrix $Z^{\mu \nu}$ has the form

$$
Z(\mathfrak{p}, \mathfrak{q})=\left(\begin{array}{cccc|c|cccc}
\mathfrak{p} & \mathfrak{q} & \ldots & \mathfrak{q} & \mathfrak{q} & \mathfrak{p q} & \ldots & \ldots & \mathfrak{p q} \\
\vdots & \ddots & \ddots & \vdots & \vdots & \vdots & \ddots & & \vdots \\
\vdots & & \ddots & \mathfrak{q} & \vdots & \vdots & & \ddots & \vdots \\
\mathfrak{p} & \ldots & \ldots & \mathfrak{p} & \mathfrak{q} & \mathfrak{p q} & \ldots & \ldots & \mathfrak{p q} \\
\hline 1 & \ldots & \ldots & 1 & 1 & \mathfrak{q} & \ldots & \ldots & \mathfrak{q} \\
\hline 1 & \ldots & \ldots & 1 & 1 & \mathfrak{p} & \mathfrak{q} & \ldots & \mathfrak{q} \\
\vdots & \ddots & & \vdots & \vdots & \vdots & \ddots & \ddots & \vdots \\
\vdots & & \ddots & \vdots & \vdots & \vdots & & \ddots & \mathfrak{q} \\
1 & \ldots & \ldots & 1 & 1 & \mathfrak{p} & \ldots & \ldots & \mathfrak{p}
\end{array}\right)
$$

where the $(d-n)$ th row and column have been marked. Omitting factors of $\mathfrak{p}$ and $\mathfrak{q}$ which can be reconstructed out of the $\mathrm{U}(1) \times \mathrm{U}(1) \times \mathrm{U}(1)_{R^{-}}$ representations by noting that the maps $\hat{\imath}(i)_{s}^{[n, a, b, r]}$ have degree $[0,0,1]$, this can again be written in a more compact form. Let $\left(f_{\mu}^{[n, a, b, r]}\right)_{\mu}$ be a basis ${ }^{15}$ of $V^{[n, a, b, r]}$ with $\left[f_{\mu}^{[n, a, b, r]}\right]=[a+\mu-d \Theta(\mu-d-n), b-\mu, r+2 \Theta(\mu-d-n)]$

\footnotetext{
${ }^{15}$ The basis used to write (4.16).
} 
and define $\hat{\tau}: f_{\mu}^{[n, a, b, r]} \mapsto f_{\mu+1 \bmod d}^{[n, a, b, r]}$, then

$$
\hat{\imath}(i)_{1}^{[n, a, b, r]}=x_{i} \mathrm{id}-y_{i} \hat{\tau}, \quad \hat{\imath}(i)_{0}^{[n, a, b, r]}=\sum_{l=0}^{d-1} A_{i}^{l}\left(x_{j}, y_{j}\right) \hat{\tau}^{l} .
$$

The GLSM matrix factorizations $\hat{I}(i)^{[n, a, b, r]}$ are labelled by representations $(a, b, r)$ of $\mathrm{U}(1) \times \mathrm{U}(1) \times \mathrm{U}(1)_{R}$ and $n \in\{0, \ldots, d-1\}$, and it makes sense to define

$$
\hat{I}^{[n+d k, a, b, r]}:=\hat{I}^{[n, a-d k, b, r+2 k]} .
$$

It is easy to see that $\hat{I}(i)^{[n, a, b, r]}$ reduces to the matrix factorization $\tilde{I}(i)^{m}$ at the Landau-Ginzburg point if

$$
(a+b-m) \bmod d=0 \quad \text { and } \quad r=\frac{2}{d}(m-a-b) .
$$

Given these lifts, one can easily lift the orbifold matrix factorization $\tilde{I}^{m}$ (c.f. (4.13)) of the identity factorization $I$ to the GLSM. Using the fact

$$
\text { Source }\left(\hat{\imath}(i)_{1}^{[n, a, b, r]}\right)=\operatorname{Target}\left(\hat{\imath}(i)_{1}^{[n+1, a+1, b, r-1]}\right)
$$

one arrives at

with

$$
\hat{I}^{[n, a, b, r]}: \hat{I}_{s}^{[n, a, b, r]} \underset{\hat{\imath}_{0}^{[n, a, b, r]}}{\stackrel{\hat{\imath}_{1}^{[n, a, b, r]}}{\rightleftarrows}} \hat{I}_{s}^{[n, a, b, r]}
$$

$$
\hat{I}_{s}^{[n, a, b, r]}=\bigoplus_{\substack{\left(s_{i}\right) \in \mathbb{Z}_{2}^{N} \\ s-\sum_{i} s_{i} \text { even }}} \bigotimes_{i=1}^{N}\left(I(i)_{s_{i}} \otimes \hat{S}\right) \otimes V^{\left[n+\sum_{i} s_{i}, a+\sum_{i} s_{i}, b, r-\sum_{i} s_{i}\right]}
$$

and

$$
\begin{aligned}
\hat{\imath}_{s}^{[n, a, b, r]}= & \sum_{\substack{\left(s_{i}\right) \in \mathbb{Z}_{2}^{N} \\
s-\sum_{i} s_{i} \text { even }}} \sum_{j=1}^{N}(-1)^{\sum_{k=1}^{j-1} s_{k}} \operatorname{id}_{I(1)_{s_{1}} \otimes \hat{S}} \otimes \ldots \\
& \ldots \otimes \hat{\imath}(j)_{s_{j}}^{\left[n+\sum_{k \neq j} s_{k}, a+\sum_{k \neq j} s_{k}, b, r-\sum_{k \neq j} s_{k}\right]} \otimes \ldots \otimes \operatorname{id}_{I(N)_{s_{N}} \otimes \hat{S}},
\end{aligned}
$$

where as before $(a+b-m) \bmod d=0$ and $r=\frac{2}{d}(m-a-b)$. Again this can be more elegantly formulated in a Koszul-type representation. For this 


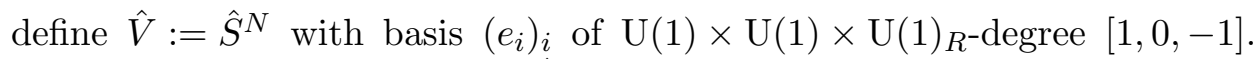
Then the matrix factorizations $\hat{I}^{[n, a, b, r]}$ can be represented as

$$
\hat{I}^{[n, a, b, r]}: \bigoplus_{s \text { odd }} \Lambda^{s} \hat{V} \otimes V^{[s+n, a, b, r]} \underset{\hat{\delta}+\hat{\sigma}}{\stackrel{\hat{\delta}+\hat{\sigma}}{\rightleftarrows}} \bigoplus_{s \text { even }} \Lambda^{s} \hat{V} \otimes V^{[s+n, a, b, r]},
$$

with

$$
\hat{\delta}=\imath_{\sum_{i} x_{i} e_{i}^{*}} \otimes \mathrm{id}-\imath_{\sum_{i} y_{i} e_{i}^{*}} \otimes \hat{\tau}, \quad \hat{\sigma}=\sum_{i, l} A_{i}^{l}\left(e_{i} \wedge \cdot\right) \otimes \hat{\tau}^{l} .
$$

Here, for ease of notation, factors of $\mathfrak{p}, \mathfrak{q}$ have been omitted. Their positions can be reconstructed out of the $\mathrm{U}(1) \times \mathrm{U}(1) \times \mathrm{U}(1)_{R}$-degrees by noting that the maps $\hat{\delta}+\hat{\sigma}$ have degree $[0,0,1]$.

The matrix factorization $\hat{I}^{[n, a, b, r]}$ has charges in the window $\mathcal{N}_{a+d-n-1} \times$ $\mathcal{N}_{b}$. Thus, the lift of the matrix factorization $\tilde{I}^{m}$ compatible with a window $\mathcal{N}_{\eta_{1}} \times \mathcal{N}_{\eta_{2}}$ is given by $\hat{I}^{\left[n, n+\eta_{1}+1-d, \eta_{2}, r\right]}$ for $n \in\{0, \ldots, d-1\}$ such that $n=$ $m-1-\eta_{1}-\eta_{2} \bmod d$.

\subsection{Transport to the large volume phase}

Having lifted the matrix factorizations $\tilde{I}^{m}$ representing the LG monodromies to the gauged linear $\sigma$-model, we can now transport them to large volume to obtain the corresponding Fourier-Mukai kernels as discussed in Section 3.5. Using the Koszul-type representation one easily sees that the large volume complex obtained by transporting the GLSM defect matrix factorization $\hat{I}^{[n, a, b, r]}$ from the Landau-Ginzburg point through phase boundary component $N_{a+d-n-1} \times N_{b}$ into the large volume phase has the form

$$
\operatorname{Cone}\left(\tilde{\mathcal{K}}_{0}^{[n, a, b, r]} \stackrel{\tilde{\sigma}}{\rightarrow} \tilde{\mathcal{C}}_{0}^{[n, a, b, r]}\right) .
$$

Here

$$
\begin{aligned}
\mathcal{K}_{0}^{[n, a, b, r]}: & \Lambda^{d-n-1} V \otimes V_{0}^{[d-1, a, b, r-d+n+1]} \stackrel{\delta}{\rightarrow} \Lambda^{d-n-2} V \otimes V_{1}^{[d-2, a, b, r-d+n+2]} \\
& \stackrel{\delta}{\rightarrow} \ldots \stackrel{\delta}{\rightarrow} \Lambda^{0} V \otimes V_{d-1}^{[n, a, b, r]}
\end{aligned}
$$

where the "unhatted" $V$ 's are defined by $V=\hat{V} \otimes_{\hat{S}} \hat{S} /\left(\mathfrak{p}, \mathfrak{q}, W\left(x_{i}\right), W\left(y_{i}\right)\right)$. For $\mu=d-n-1, V_{\mu}^{[n, a, b, r]}=\operatorname{spann}_{R}\left(f_{\nu}^{[n, a, b, r]}\right)_{0 \leq \nu \leq \mu}$ are the subspaces of 
$\hat{V}^{[n, a, b, r]}$ of $\mathrm{U}(1)_{R^{-c h a r g e}} r$. In more detail this complex looks like

$$
\begin{array}{ccccccc}
\Lambda^{d-n-1} V[a, b] & \rightarrow & \Lambda^{d-n-2} V[a, b] & \rightarrow & \cdots & \rightarrow & \Lambda^{0} V[a, b] \\
& \searrow & \oplus & \searrow & \cdots & \searrow & \oplus \\
& \Lambda^{d-n-2} V[a+1, b-1] & \rightarrow & \cdots & \rightarrow & \Lambda^{0} V[a+1, b-1] \\
& & & & & \oplus \\
& & & & \cdots & \Lambda^{0} V[a+d-n-1 \\
& & & & & b-d+n+1]
\end{array}
$$

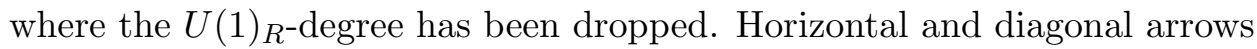
represent the maps $\imath_{\sum_{j} x_{j} e_{j}^{*}}$ and $\imath_{\sum_{j} y_{j} e_{j}^{*}}$, respectively. As discussed in Section 3.4 the sheafifications of the rows in this complex are quasi-isomorphic to

$$
\left.\Omega_{\mathbb{P}^{N-1}}^{d-n-1-i}(-a-i)\right|_{X} \otimes \mathcal{O}_{X}(-b+i)\{-r+d-n-1-i\},
$$

where $X$ is the projective hypersurface $\{W=0\}$ in $\mathbb{P}^{N-1}$. The sheafification $\tilde{\mathcal{K}}_{0}^{[n, a, b, r]}$ can therefore be written as

$$
\begin{gathered}
\left.\left.\Omega_{\mathbb{P}^{N-1}}^{d-n-1}(-a)\right|_{X} \otimes \mathcal{O}_{X}(-b) \rightarrow \Omega_{\mathbb{P}^{N-1}}^{d-n-2}(-a-1)\right|_{X} \otimes \mathcal{O}_{X}(-b+1) \rightarrow \ldots \\
\left.\ldots \rightarrow \Omega_{\mathbb{P}^{N-1}}^{0}(-a-d+n+1)\right|_{X} \otimes \mathcal{O}_{X}(-b+d-n-1),
\end{gathered}
$$

where the last term is at position $r$.

To discuss the complexes $\mathcal{C}_{0}^{[n, a, b, r]}$, define $V_{\mu}^{[n, a, b, r]}$ for all $\mu$ to be the subspace of $\hat{V}^{[n, a, b, r]}$ of $\mathrm{U}(1)_{R^{-c h a r g e ~}} r+2((\mu+n) \operatorname{div} d)$, and denote its basis vectors by $f_{\nu}^{[n, a, b, r]}=\mathfrak{p}^{(\mu+n) \operatorname{div} d-(\nu+n) \operatorname{div} d} \mathfrak{q}^{\nu \operatorname{div} d} f_{\nu \bmod d}^{[n, a, b, r]}$.

Indeed, as in the discussion of the tensor product B-branes in Section 3.4 the complex $\mathcal{C}_{0}^{[n, a, b, r]}$ can be written as successive cone:

$$
\mathcal{C}_{i}^{[n, a, b, r]}=\operatorname{Cone}\left(\mathcal{K}_{i+1}^{[n, a, b, r]} \stackrel{\sigma}{\rightarrow} \mathcal{C}_{i+1}^{[n, a, b, r]}\right),
$$

where

$$
\mathcal{K}_{i+1}^{[n, a, b, r]}=(\mathfrak{p}+\mathfrak{q}) \mathcal{K}_{i}^{[n, a, b, r]} \quad \text { and } \quad \mathcal{C}_{i+1}^{[n, a, b, r]}=(\mathfrak{p}+\mathfrak{q}) \mathcal{C}_{i}^{[n, a, b, r]} \quad \text { for } i>0
$$

and $\mathcal{C}_{i}^{[n, a, b, r]}$ is an infinite complex which starts at position $r-d+2+2 i$. Moreover,

$$
\begin{gathered}
\mathcal{K}_{1}^{[n, a, b, r]}: \Lambda^{d} V \otimes V_{d-n}^{[n+d, a, b, r-d]} \stackrel{\delta}{\rightarrow} \Lambda^{d-1} V \otimes V_{d-n+1}^{[n+d-1, a, b, r-d+1]} \stackrel{\delta}{\rightarrow} \ldots \\
\ldots \stackrel{\delta}{\rightarrow} \Lambda^{i} V \otimes V_{2 d-i-n}^{[n+i, a, b, r-i]} \stackrel{\delta}{\rightarrow} \Lambda^{0} V \otimes V_{2 d-n}^{[n, a, b, r]} .
\end{gathered}
$$


Similarly to $\mathcal{K}_{0}^{[n, a, b, r]}$, also $\mathcal{K}_{1}^{[n, a, b, r]}$ can be written in triangular shape (4.31), and its sheafification $\tilde{\mathcal{K}}_{1}^{[n, a, b, r]}$ is quasi-isomorphic to

$$
\begin{aligned}
& \left.\Omega_{\mathbb{P}^{N-1}}^{d-1}(-a+n)\right|_{X} \otimes \mathcal{O}_{X}(-b+d-n) \rightarrow \\
& \left.\quad \rightarrow \Omega_{\mathbb{P}^{N-1}}^{d-2}(-a+n-1)\right|_{X} \otimes \mathcal{O}_{X}(-b+d-n+1) \rightarrow \cdots \\
& \left.\quad \cdots \rightarrow \Omega_{\mathbb{P}^{N-1}}^{0}(-a+n-d+1)\right|_{X} \otimes \mathcal{O}_{X}(-b+2 d-n-1),
\end{aligned}
$$

where the last term is at position $r+2$. Indeed, $\tilde{\mathcal{K}}_{i}^{[n, a, b, r]}$ for any $i \geq 0$ is quasi-isomorphic to the complex (4.37) tensored by $\mathcal{O}_{X} \otimes \mathcal{O}_{X}(d i)$ and shifted to the right by $2 i$.

Using Beilinson's resolution

$$
\begin{aligned}
0 \rightarrow \Omega_{\mathbb{P}^{N-1}}^{N-1}(N-1) \otimes \mathcal{O}_{\mathbb{P}^{N-1}}(1-N) & \rightarrow \ldots \rightarrow \Omega_{\mathbb{P}^{N-1}}^{1}(1) \otimes \mathcal{O}_{\mathbb{P}^{N-1}}(-1) \\
& \rightarrow \Omega_{\mathbb{P}^{N-1}}^{0}(0) \otimes \mathcal{O}_{\mathbb{P}^{N-1}}(0) \rightarrow \mathcal{O}_{\Delta} \rightarrow 0
\end{aligned}
$$

of the diagonal $\Delta \subset \mathbb{P}^{N-1} \times \mathbb{P}^{N-1}$, we easily see that

$$
\begin{aligned}
\tilde{\mathcal{K}}_{i}^{[n, a, b, r]} & \cong \mathcal{O}_{\Delta} \otimes\left(\mathcal{O}_{X}(-a+n-d+1)\right. \\
& \left.\otimes \mathcal{O}_{X}(-b+d-n-1+d i)\right)\{-r-2 i\} .
\end{aligned}
$$

for $i>0$. One can then use the resolution

$$
\begin{aligned}
0 & \rightarrow \mathcal{O}_{\mathbb{P}^{N-1}}(-d) \otimes \mathcal{O}_{\mathbb{P}^{N-1}}(-d) \\
& \rightarrow\left(\mathcal{O}_{\mathbb{P}^{N-1}}(-d) \otimes \mathcal{O}_{\mathbb{P}^{N-1}}\right) \oplus\left(\mathcal{O}_{\mathbb{P}^{N-1}} \otimes \mathcal{O}_{\mathbb{P}^{N-1}}(-d)\right) \\
& \rightarrow \mathcal{O}_{\mathbb{P}^{N-1}} \otimes \mathcal{O}_{\mathbb{P}^{N-1}} \rightarrow \mathcal{O}_{X \times X} \rightarrow 0
\end{aligned}
$$

of $\mathcal{O}_{X} \otimes \mathcal{O}_{X}$ on $\mathbb{P}^{N-1} \times \mathbb{P}^{N-1}$ to obtain

$$
\begin{aligned}
\tilde{\mathcal{K}}_{i}^{[n, a, b, r]} \cong & \mathcal{O}_{\Delta X}(-a-b+(i-1) d)\{-r-2 i+1\} \\
& \oplus \mathcal{O}_{\Delta X}(-a-b+d i)\{-r-2 i\}
\end{aligned}
$$

for $i>0$. In this complex all maps are zero, i.e., $\tilde{\mathcal{K}}_{i}^{[n, a, b, r]}$ is quasi-isomorphic to its cohomology. Now, it is not difficult to see that the map $\sigma: \tilde{\mathcal{K}}_{i}^{[n, a, b, r]} \rightarrow$ $\tilde{\mathcal{K}}_{i+1}^{[n, a, b, r]}$ in cohomology descends to the map $1: \mathcal{O}_{\Delta X}(-a+b+d i)\{-r-$ $2 i\} \rightarrow \mathcal{O}_{\Delta X}(-a+b+d i)\{-r-2 i\}$ from the second summand in (4.41) for 
$\tilde{\mathcal{K}}_{i}^{[n, a, b, r]}$ to the first summand for $\tilde{\mathcal{K}}_{i+1}^{[n, a, b, r]}$. Thus these summands successively cancel in the cone construction of $\tilde{\mathcal{C}}_{0}^{[n, a, b, r]}$, and one ends up with

$$
\tilde{\mathcal{C}}_{0}^{[n, a, b, r]} \cong \mathcal{O}_{\Delta X}(-a-b)\{-r-1\}
$$

Thus, this indeed completes (4.33) to the large volume kernel

$$
\begin{aligned}
& \left.\left.\Omega_{\mathbb{P}^{N-1}}^{d-n-1}(-a)\right|_{X} \otimes \mathcal{O}_{X}(-b) \rightarrow \Omega_{\mathbb{P}^{N-1}}^{d-n-2}(-a-1)\right|_{X} \otimes \mathcal{O}_{X}(-b+1) \rightarrow \cdots \\
& \left.\quad \cdots \rightarrow \Omega_{\mathbb{P}^{N-1}}^{0}(-a-d+n+1)\right|_{X} \otimes \mathcal{O}_{X}(-b+d-n-1) \rightarrow \mathcal{O}_{\Delta X}(-a-b),
\end{aligned}
$$

where the last term is at position $r+1$.

Let us transport the LG-monodromy defect matrix factorizations $\tilde{I}^{m}$ from the Landau-Ginzburg point through the phase boundary component $N_{0} \times$ $N_{d-1}$ corresponding to the combination of dual charge windows $\mathcal{N}_{0} \times\left(-\mathcal{N}_{0}\right)$ into the large volume phase. To simplify notation we parametrize $m=M+$ $d k$ with $M \in\{-d, \ldots,-1\}$. As discussed at the end of Section 4.2, the lift of the matrix factorization $\tilde{I}^{m}$ to this window is given by $\hat{I}^{[M+d, M+1, d-1,2 k-2]}$. Following the arguments above, the corresponding large volume complex can be read off from (4.43) to be

$$
\begin{aligned}
& \left(\left.\Omega_{\mathbb{P}^{N-1}}^{-M-1}(-M-1)\right|_{X} \otimes \mathcal{O}_{X}(1-d)\right. \\
& \left.\quad \rightarrow \Omega_{\mathbb{P}^{N-1}}^{-M-2}(-M-2)\right|_{X} \otimes \mathcal{O}_{X}(2-d) \rightarrow \cdots \\
& \left.\left.\quad \cdots \rightarrow \Omega_{\mathbb{P}^{N-1}}^{0}(0)\right|_{X} \otimes \mathcal{O}_{X}(-M-d) \rightarrow \mathcal{O}_{\Delta X}(-M-d)\right)\{1-2 k\}
\end{aligned}
$$

As discussed in Section 3 the associated Fourier-Mukai kernels are obtained by tensoring these complexes by $\mathcal{O}_{X} \otimes \mathcal{O}_{X}(d)\{-1\}$ :

$$
\begin{gathered}
\left(\left.\left.\Omega_{\mathbb{P}^{N-1}}^{-M-1}(-M-1)\right|_{X} \otimes \mathcal{O}_{X}(1) \rightarrow \Omega_{\mathbb{P}^{N-1}}^{-M-2}(-M-2)\right|_{X} \otimes \mathcal{O}_{X}(2) \rightarrow \ldots\right. \\
\left.\left.\quad \cdots \rightarrow \Omega_{\mathbb{P}^{N-1}}^{0}(0)\right|_{X} \otimes \mathcal{O}_{X}(-M) \rightarrow \mathcal{O}_{\Delta X}(-M)\right)\{-2 k\}
\end{gathered}
$$

We indeed find the expected (see $[14,16,30]$ ) Fourier-Mukai kernels for the Landau-Ginzburg monodromies $G^{-m}$, where $G$ is the generator of this monodromy (cf. table 2). In particular for the identity defect $\tilde{I}^{0}$, the resulting kernel $(M=-d, k=1)$ is nothing but $\mathcal{O}_{\Delta X}$, the kernel of the trivial Fourier-Mukai transform. 
Table 2: Summary of the result of the transport of the Landau-Ginzburg monodromy defects given in the first column through the phase boundary component $N_{0} \times\left(-N_{0}\right)$ into the large volume phase. The second column contains the lifts of the defects to the gauged linear $\sigma$-model compatible with the corresponding charge window $\mathcal{N}_{0} \times\left(-\mathcal{N}_{0}\right)$. The last column shows the resulting Fourier-Mukai kernels which are obtained from the large volume defects by tensoring with $\mathcal{O}_{X} \otimes \mathcal{O}_{X}(d)\{1\}$. The underlined sheaves are at position zero in the complexes.

\begin{tabular}{lll}
\hline LG defect & GLSM defect & FM kernel \\
\hline$\vdots$ & $\vdots$ & $\vdots$ \\
$\tilde{I}^{0}$ & $\hat{I}^{[0,1-d, d-1,0]}$ & $\underline{\mathcal{O}_{\Delta X}}$ \\
$\tilde{I}^{-1}$ & $\hat{I}^{[d-1,0, d-1,-2]}$ & $\mathcal{O}_{X} \otimes \mathcal{O}_{X}(1) \rightarrow \underline{\mathcal{O}_{\Delta X}(1)}$ \\
$\tilde{I}^{-2}$ & $\hat{I}^{[d-2,-1, d-1,-2]}$ & $\left.\Omega_{\mathbb{P}^{N-1}}^{1}(1)\right|_{X} \otimes \mathcal{O}_{X}(1) \rightarrow \mathcal{O}_{X} \otimes \mathcal{O}_{X}(2) \rightarrow \underline{\mathcal{O}_{\Delta X}(2)}$ \\
$\vdots$ & $\vdots$ & $\vdots$ \\
$\tilde{I}^{-(d-1)}$ & $\hat{I}^{[1,-(d-2), d-1,-2]}$ & $\left.\Omega_{\mathbb{P}^{N-1}}^{d-2}(d-2)\right|_{X} \otimes \mathcal{O}_{X}(1) \rightarrow \mathcal{O}_{X} \otimes \mathcal{O}_{X}(d-1)$ \\
$\tilde{I}^{-d}$ & $\hat{I}^{[0,1-d, d}(d-1)$ & $\underline{\mathcal{O}_{\Delta X}(d-1)}$ \\
$\vdots$ & $\vdots$ & $\mathcal{O}_{\Delta X}\{2\}$ \\
\hline
\end{tabular}

\subsection{Conifold and large-volume monodromies in the GLSM moduli space}

Before we start to analyse the monodromy structure in the gauged linear $\sigma$-model we should remark that the Kähler moduli space of the latter not always coincides with the Kähler moduli space of the corresponding nonlinear $\sigma$-model. Already in its geometric phases gauged linear $\sigma$-models only capture those Kähler moduli of the non-linear $\sigma$-models on hyperplanes $X$, which are inherited from the ambient space. ${ }^{16}$ However, if the ambient space is the projective space $\mathbb{P}^{N-1}$, and if $N>4$ then the Lefschetz hyperplane theorem guarantees that the pullback of the embedding map induces an isomorphism from the cohomology group $H^{2}\left(\mathbb{P}^{N-1}, \mathbb{Z}\right)$ of the ambient space to the cohomology group $H^{2}(X, \mathbb{Z})$ of the embedding space. Therefore, for

\footnotetext{
${ }^{16}$ In particular for more general geometries this implies that one cannot include nontoric divisors because the gauged linear $\sigma$-model is tailor-made to describe only toric data.
} 
these cases we expect that the Kähler moduli spaces of gauged linear $\sigma$-model and non-linear $\sigma$-model and also monodromies in these moduli spaces agree.

Note that the above argument excludes the cubic torus in $\mathbb{P}^{3}$ and the quartic $\mathrm{K} 3$ surface in $\mathbb{P}^{4}$. In the former example, although the dimensions of the integer cohomology groups are the same, the pullback of the generator of $H^{2}\left(\mathbb{P}^{3}, \mathbb{Z}\right)$ does not map to the generator of $H^{2}(X, \mathbb{Z})$. As a consequence the large radius monodromy of the gauged linear $\sigma$-model is the third power of the large radius monodromy of the non-linear $\sigma$-model on the torus [31], whereas monodromies around Landau-Ginzburg and conifold point agree. In the latter example the gauged linear $\sigma$-model description only covers a slice of the non-linear $\sigma$-model Kähler moduli space, because for (algebraic) K3 surfaces $\operatorname{dim} H^{1,1}(X)=20$.

After this interlude about the relation between Kähler moduli spaces and monodromies in non-linear and gauged linear $\sigma$-models, let us now return to the discussion of the monodromies in gauged linear $\sigma$-models. By transporting the LG monodromy defects through different phase boundary components in the Kähler moduli space of the gauged linear $\sigma$-model, one can indeed also obtain the monodromies around conifold and large volume point in the gauged linear $\sigma$-model.

For instance, transporting the identity defect $\tilde{I}^{0}$ from the LandauGinzburg point into the large volume phase through the component $N_{0} \times N_{d}$ of the phase boundary is the same as first transporting it through the component $N_{0} \times N_{d-1}$ and then transporting it around the conifold point once. As discussed in Section 4.3, transporting the identity defect through $N_{0} \times N_{d-1}$ one obtains the large volume kernel $\mathcal{O}_{\Delta X}$ of the identity Fourier-Mukai transform tensored by $\mathcal{O}_{X} \otimes \mathcal{O}_{X}(-d)\{1\}$. The effect of taking it around the conifold point is then given by an action of the Fourier-Mukai transformation associated to the conifold monodromy. Therefore, transporting the identity defect through $N_{0} \times N_{d}$ gives rise the the Fourier-Mukai kernel of the conifold monodromy tensored by $\mathcal{O}_{X} \otimes \mathcal{O}_{X}(-d)\{1\}$.

As has been shown in (4.2), the lift of the identity defect $\tilde{I}^{0}$ to the GLSM compatible with $\mathcal{N}_{0} \times \mathcal{N}_{d}$, i.e., the one which can be transported through the phase boundary component $N_{0} \times N_{d}$ is given by $\hat{I}^{[d-1,0, d,-2]}$. The corresponding large volume complex can be read off from (4.43) to be

$$
\left(\mathcal{O}_{X} \otimes \mathcal{O}_{X}(-d) \rightarrow \mathcal{O}_{\Delta X}(-d)\right)\{1\},
$$

giving rise to the expected Fourier-Mukai kernel

$$
\mathcal{O}_{X} \otimes \mathcal{O}_{X} \rightarrow \mathcal{O}_{\Delta X}
$$


associated to the conifold monodromy ${ }^{17}$ (see $[14,16,30]$ for a target space geometric derivation of this monodromy transformation).

The Landau-Ginzburg realization of the conifold monodromy can be obtained by transporting the large volume complex (4.46) back to the Landau-Ginzburg point through the phase boundary component $N_{0} \times N_{d-1}$ corresponding to the charge window $\mathcal{N}_{0} \times\left(-\mathcal{N}_{0}\right)$. This is easy to accomplish. We first note that (4.46) is a cone of two one-term complexes which we know how to transport to the LG point. Namely, $\mathcal{O}_{\Delta X}(-d)\{1\}$ has been obtained by transporting $\tilde{I}^{0}$ through $N_{0} \times N_{d-1}$ to large volume in Section 4.3 above. Moreover, $\mathcal{O}_{X} \otimes \mathcal{O}_{X}(-d)\{1\}$ is a product sheaf. As has been discussed in Section 3.4, the first factor $\mathcal{O}_{X}$ can be obtained by transporting the tensor product matrix factorization $\tilde{P}^{0}$ of $W\left(x_{i}\right)$ to large volume through $N_{0}$.

Furthermore, using the fact (discussed in Appendix A) that the dual $P^{*}$ of a matrix factorization $P$ transported to large volume through a phase boundary component $N$ corresponding to charge window $\mathcal{N}$ gives rise to the dual of the large volume complex obtained by transporting $P$ to large volume through the component $-N$ associated to the dual charge window $-\mathcal{N}$ tensored by $\mathcal{O}_{X}(-d)\{1\}$, we see that $\mathcal{O}_{X}(-d)\{1\} \cong \mathcal{O}_{X}^{*}(-d)\{1\}$ can be obtained by transporting $\left(\tilde{P}^{0}\right)^{*}$ to large volume through phase boundary component $N_{d-1}$. Thus, the realisation of the conifold monodromy is given by the cone

$$
\text { Cone }\left(\tilde{r}: \tilde{P}^{0}(x) \otimes\left(\tilde{P}^{0}(y)\right)^{*} \rightarrow \tilde{I}^{0}\right),
$$

where $\tilde{P}^{0}(x)$ denotes the respective equivariant tensor product matrix factorization of $W\left(x_{1}, \ldots, x_{N}\right)$, and the map $\tilde{r}$ is induced by the identity map id : $P^{0} \rightarrow P^{0}$ by means of the folding isomorphism (3.31). In physics terminology this is the tachyon condensation of the sum of defect matrix factorizations

$$
\tilde{P}^{0}(x) \otimes\left(\tilde{P}^{0}(y)\right)^{*}\{1\} \oplus \tilde{I}^{0}
$$

with tachyon associated to $\tilde{r}$. In Section 5 we will discuss the conifold monodromies in more detail and more generality.

To obtain the inverse of the large volume monodromy one has to compose the generator of the conifold monodromy with the inverse of the generator $G$ of the Landau-Ginzburg monodromy. The corresponding large volume complex can be obtained by transporting the defect $\tilde{I}^{1}$ through the phase

\footnotetext{
${ }^{17}$ This is indeed also the expected monodromy in the Kähler moduli space of the corresponding non-linear $\sigma$-model.
} 
boundary component $N_{0} \times N_{d}$. The lift of $\tilde{I}^{1}$ compatible with the corresponding charge window $\mathcal{N}_{0} \times\left(-\mathcal{N}_{0}+1\right)$ is given by $\hat{I}^{[0,1-d, d, 0]}$. The large volume complex can then be read off from (4.43) to be

$$
\mathcal{O}_{\Delta X}(-1-d)\{1\}
$$

Tensoring by $\mathcal{O}_{X} \otimes \mathcal{O}_{X}(d)\{-1\}$ we obtain the Fourier-Mukai kernel $\mathcal{O}_{\Delta X}(-1)$ of the inverse of the large-volume monodromy. This is also the expected result for the monodromy around the large volume limit in the Kähler moduli space of the gauged linear $\sigma$-model $[13,14,16]$. Note that, as alluded to above, in the case that $X$ is the cubic torus in $\mathbb{P}^{2}$, i.e., $N=d=3$, this monodromy does not coincide with the large volume monodromy in the Kähler moduli space of the corresponding non-linear $\sigma$-model, but is its third power.

\section{Conifold monodromy}

Let us now discuss the conifold monodromy in more detail. A conifold point in the Kähler moduli space of a non-linear $\sigma$-model on a Calabi-Yau manifold $X$ is characterized by the property that at this point a certain B-type BPS brane $Q$ becomes massless. This means that its (quantum) world-volume in the Calabi-Yau space vanishes while the (quantum) worldvolumes of other B-type branes remain finite [50]. Such points are easily identified in the mirror geometry, where the vanishing quantum cycle of the B-brane $Q$ arises classically as a singularity in the mirror Calabi-Yau manifold. Locally, the latter can be described by the geometry of a singular conifold [51].

B-type D-branes transform under the monodromy around conifold points in Kähler moduli space in a non-trivial way. The monodromy action on D-brane charges is encoded in the periods in the vicinity of the conifold point $[50,52,53]$. But the analysis can be extended beyond the level of Dbrane charges to the category of topological B-branes. It turns out that the conifold monodromy acts on the large volume realization $D^{b}(X)$ of this category by means of the Fourier-Mukai transformation $\Phi^{\mathcal{K}_{\mathcal{Q}}^{\mathrm{C}}}$ with kernel

$$
\mathcal{K}_{\mathcal{Q}}^{\mathrm{C}}=\operatorname{Cone}\left(r: \mathcal{Q} \otimes \mathcal{Q}^{\vee} \rightarrow \mathcal{O}_{\Delta X}\right)
$$

determined by the large volume complex $\mathcal{Q}$ associated to the B-brane $Q$ $[14,54]$. Here $\mathcal{Q}^{\vee}$ denotes the dual of $\mathcal{Q}$, and $\mathcal{O}_{\Delta X}$ is structure sheaf of the diagonal $\Delta X \subset X \times X$. The map $r$ is the restriction map to the diagonal 
$\Delta X$. If for instance $\mathcal{Q}=\mathcal{O}_{X}$, then the map $r$ restricts $\mathcal{O}_{X \times X}=\mathcal{O}_{X} \otimes \mathcal{O}_{X}$ to $\mathcal{O}_{\Delta X}$.

In general, the map $r$, which is an element of $\operatorname{Hom}\left(\mathcal{Q} \otimes \mathcal{Q}^{\vee}, \mathcal{O}_{\Delta X}\right)$, is induced from the identity map id $\in \operatorname{Hom}(\mathcal{Q}, \mathcal{Q})$ by means of the following chain of isomorphisms: The complex $\mathcal{Q}$ is quasi-isomorphic to its image $\Phi^{\mathcal{O}_{\Delta x}}(\mathcal{Q})$ under the trivial Fourier-Mukai transform. Therefore $\operatorname{Hom}(\mathcal{Q}, \mathcal{Q})$ is isomorphic to $\operatorname{Hom}\left(\mathcal{Q}, \Phi^{\mathcal{O}_{\Delta x}}(\mathcal{Q})\right)$, which in turn is isomorphic to $\operatorname{Hom}(\mathcal{Q} \otimes$ $\left.\mathcal{Q}^{\vee}, \mathcal{O}_{\Delta X}\right)$ (cf. Equation (3.30)).

The action of the Fourier-Mukai transformation $\Phi^{\mathcal{K}_{\mathcal{Q}}^{\mathrm{C}}}$ associated to the kernel (5.1) on a complex $\mathcal{E}$ can also be represented in the following way $[31,54]$ :

$$
\mathcal{E} \mapsto \text { Cone }(\text { ev }: \operatorname{Hom}(\mathcal{Q}, \mathcal{E}) \otimes \mathcal{Q} \rightarrow \mathcal{E})
$$

where 'ev' denotes the evaluation map. This expression also makes more transparent that the advocated Fourier-Mukai transformation $\Phi^{\mathcal{K}_{\mathcal{Q}}^{\mathrm{C}}}$ indeed reduces to the expected map on the level of D-brane charges.

In this paper, we focused our considerations on non-linear $\sigma$-models whose target spaces $X$ are projective Calabi-Yau hypersurfaces. The Kähler moduli spaces of the gauged linear $\sigma$-model realization of these models exhibit a conifold singularity, at which the (quantum) world-volume of the entire target space is zero.

The monodromy around this point was derived in Section 4.4 by lifting the identity defect to the gauged linear $\sigma$-model and transporting it around the singularity. In the large volume phase we indeed obtained the FourierMukai transformation with kernel (4.47) which is of the general form $\mathcal{K}_{\mathcal{O}}^{\mathrm{C}}$ where $\mathcal{Q}=\mathcal{O}_{X}$ is the large volume complex representing the B-brane which becomes massless at the conifold point.

Thus, this approach provides an independent derivation of the conifold monodromy for this particular case. Moreover, it also yields a realization of this monodromy at the Landau-Ginzburg point. Namely, we obtained the cone (4.48) as the matrix factorization representing the defect which realizes this monodromy. The structure of this matrix factorization is very reminiscent of the structure of the Fourier-Mukai kernel $\mathcal{K}_{\mathcal{Q}}^{\mathrm{C}}$. In the following, we will generalize these Landau-Ginzburg defects to arbitrary B-branes $Q$ and show that transported to large volume they reproduce the Fourier-Mukai kernels $\mathcal{K}_{\mathcal{Q}}^{\mathrm{C}}$. Hence, we obtain a Landau-Ginzburg realization of arbitrary conifold monodromies. 


\subsection{Conifold-like defects in general CFTs}

As preparation, we would like to give a non-technical description of a class of defects arising in any $N=(2,2)$ superconformal field theory, whose action on boundary conditions mimics conifold monodromy transformations. The conifold monodromy defects which we will present in the next section are a concrete example of this general construction in the context of LandauGinzburg models.

As described in the Introduction, conifold monodromies can be understood as follows. Any number of D-branes $Q$ which become massless at a conifold point can be created there at no cost in energy. When one transports a massive probe D-brane $E$ around this point, it forms bound states with these D-branes, provided there are suitable open string tachyons between $Q$ and $E$. This is exactly what is described by formula (5.2) in the context of large volume non-linear $\sigma$-models.

Therefore, a defect mimicking such a monodromy transformation has to map a boundary condition $E$ to itself, and in addition it has to create a copy of a chosen boundary condition $Q$ for every possible tachyon between $Q$ and $E$. Moreover, it has to form a bound state of this collection of boundary conditions.

Defects which upon fusion with a boundary condition $E$ create the above constituent boundary conditions before bound state formation are very easy to find. First of all, the identity defect Id, which exists in all CFTs maps any boundary condition $E$ to itself. The second constituent can be obtained by fusion with a totally reflective defect.

Totally reflective defects are defects which do not allow the transmission of excitation from one of the adjacent CFTs to the other. Hence, they are defined by boundary conditions in each of these CFTs. For instance, one can choose the boundary condition $Q$ on one side of the defect and the worldsheet parity dual $Q^{*}$ of $Q$ on the other side. If the corresponding defect $T_{Q}=Q \otimes Q^{*}$ is fused with the boundary condition $E$, it creates a copy of the boundary condition $Q$ for every tachyon between $Q$ and $E$, or to be more precise, it turns $E$ into the boundary condition $\mathcal{H}^{*}(Q, E) \otimes Q$. Thus, identity defect $\mathrm{Id}$ and the totally reflective defect $T_{Q}$ indeed provide the building blocks needed to produce conifold-like transformation on boundary conditions, cf. (5.2).

The bound state formation of this collection of boundary conditions is then induced by a bound state formation of the anti-defect $T_{Q}\{1\}$ whose R-charges have been shifted by 1 relative to $T_{Q}$ and the trivial defect Id. 

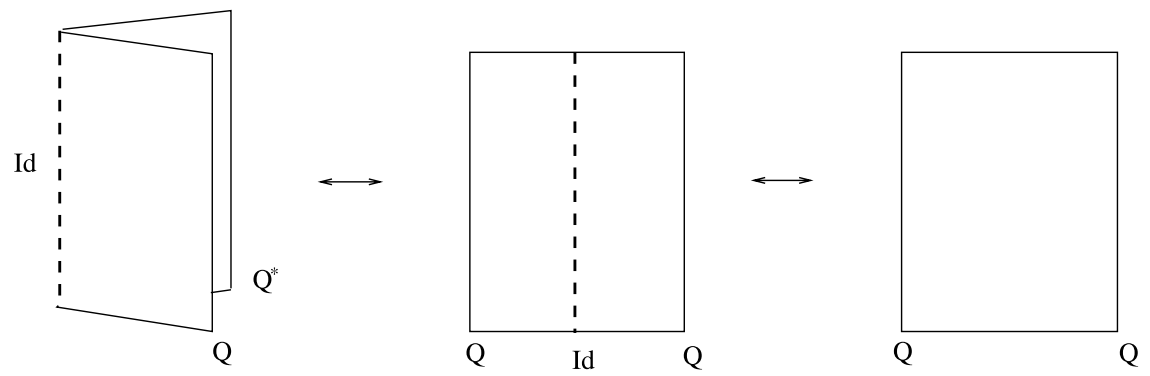

Figure 5: The spectrum of boundary fields of a boundary condition $Q$ (right) always contains the identity. This yields a universal element in the spectrum of defect-changing fields between the totally reflective defect $T_{Q}$ and the identity defect (left).

Indeed, there is a universal defect-changing field in the spectrum between these two defects which can be used to trigger the corresponding tachyon condensation. It can be described by means of the folding trick, cf. figure 5 . Being a totally reflective defect, one can cut open the world-sheet along the defect $T_{Q}$. In particular, if Id and $T_{Q}$ run parallel along a cylinder, the latter can be unfolded to a strip with boundary conditions $Q$ at both its edges. Since Id is the trivial defect, the spectrum of defect-changing fields between Id and $T_{Q}$ is therefore isomorphic to the spectrum of boundary fields on the boundary condition $Q$. (This is a special case of the folding formula (3.31).)

Thus, the identity boundary field on $Q$ gives rise to a universal bosonic defect-changing field between $T_{Q}$ and $\mathrm{Id}$, which is promoted to a fermionic field between the shifted defect $T_{Q}\{1\}$ and Id. This defect-changing field can be used to form a bound state $C_{Q}$ of the defects $T_{Q}\{1\}$ and Id, and upon fusion of $C_{Q}$ with a boundary condition $E$ it induces exactly the tachyons between $E$ and the collection of boundary conditions $Q$ created by $T_{Q}$ (i.e., $\left.\mathcal{H}^{*}(Q, E) \otimes Q\right)$, which trigger the desired bound state formation. It is the exact analogue of the map ev in (5.2).

Hence, the bound state defect $C_{Q}$ realizes conifold-like actions on boundary conditions in any $N=(2,2)$ superconformal field theory.

To relate this general construction to the derivation of the special conifold defects in Section 4.4, note that the conifold defects (4.48) obtained there in the context of Landau-Ginzburg orbifolds indeed are of the form $C_{Q}$, where $Q$ is given by the matrix factorization $\tilde{P}^{0}$. The map $\tilde{r}$ is exactly the universal defect-changing field discussed above. 


\subsection{Conifold defects in the Landau-Ginzburg phase}

After this digression into the context of general $N=(2,2)$ superconformal field theories, we will turn back to conifold monodromies in non-linear $\sigma$-models. In the following, we will realize the Fourier-Mukai transformations $\Phi^{\mathcal{K}_{\mathcal{Q}}^{\mathrm{C}}}$ representing monodromies around conifold points associated to B-type D-branes with arbitrary large volume complex $\mathcal{Q}$ as defects in the Landau-Ginzburg phase.

Guided by the above discussion, we first need to identify the equivariant matrix factorization

$$
Q: Q_{1} \underset{q_{0}}{\stackrel{q_{1}}{\rightleftarrows}} Q_{0},
$$

of $W\left(x_{1}, \ldots, x_{N}\right)$ which in the Landau-Ginzburg phase represents the BPS brane that becomes massless at the chosen conifold locus. Let $Q^{*}$ be the dual of the matrix factorization $Q$ defined as in (3.32). Note that the representations of the orbifold group $\Gamma$ and $R$-symmetry group on the dual matrix factorization $Q^{*}$ are given by the dual of the representations on $Q$.

Now let us denote by $Q(y)$ the matrix factorization $W\left(y_{1}, \ldots, y_{N}\right)$ obtained from $Q$ by replacing the variables $x_{i}$ by $y_{i}$, and set $Q(x)=Q$. The tensor product (as defined in (2.3)) $Q(x) \otimes Q^{*}(y)$ of the matrix factorizations $Q(x)$ and the dual of $Q(y)$ yields a matrix factorization of $W\left(x_{1}, \ldots, x_{N}\right)-$ $W\left(y_{1}, \ldots, y_{N}\right)$ which will eventually corresponds to the large volume complex $\mathcal{Q} \otimes \mathcal{Q}^{\vee}$ in the conifold Fourier-Mukai kernel $\mathcal{K}_{\mathcal{Q}}^{\mathrm{C}}$ defined in (5.1). We denote this tensor product matrix factorization by $T_{Q}$. Concretely, it reads

$$
\begin{aligned}
& T_{Q}=Q(x) \otimes Q^{*}(y):
\end{aligned}
$$

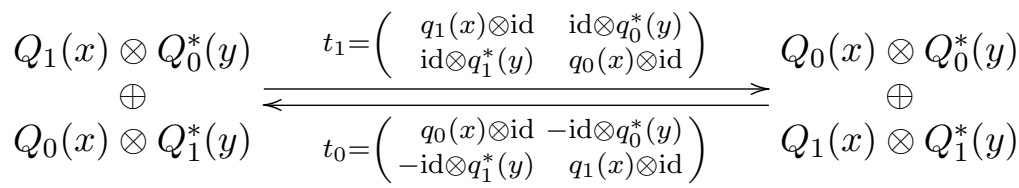

This is a $\Gamma \times \Gamma \times \mathrm{U}(1)_{R^{-}}$equivariant matrix factorization with representations induced by the ones on the factors $Q(x)$ and $Q^{*}(y)$.

The next ingredient in the defect (4.48) realizing the monodromy around the conifold point associated to $\mathcal{O}_{X}$ is the matrix factorization $\tilde{I}^{0}$ representing the identity defect. As has been discussed in Section 4.1 this corresponds to the trivial Fourier-Mukai transform at large volume, which has the diagonal structure sheaf $\mathcal{O}_{\Delta X}$ as kernel. 
Finally, the map $\tilde{r}$ in (4.48) is replaced by the analogous map $\tilde{r} \in \mathcal{H}_{\text {orb }}^{0}\left(T_{Q}\right.$, $\left.\tilde{I}^{0}\right)$ represented by the maps $\tilde{r}_{s}$ in the commutative diagram

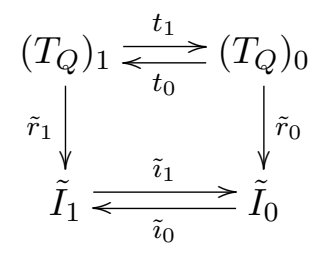

which is induced by the identity morphism of the matrix factorization $Q$ by means of the folding isomorphisms $\mathcal{H}^{0}(Q(x), Q(x)) \cong \mathcal{H}^{0}\left(Q(x), \tilde{I}^{0} * Q(y)\right) \cong$ $\mathcal{H}^{0}\left(T_{Q}, \tilde{I}^{0}\right)$, cf. (3.31). This map corresponds to the restriction map $r$ in the Fourier-Mukai kernel (5.1) at large volume. Its explicit computation is given in Appendix B.

Now we have all the ingredients at hand to state the matrix factorization representing the the conifold defect at the Landau-Ginzburg point. As in (4.48) it is given by the cone construction with respect to the map $\tilde{r}$ :

$$
C_{Q}=\operatorname{Cone}\left(\tilde{r}: Q(x) \otimes Q^{*}(y) \rightarrow \tilde{I}^{0}\right) .
$$

In physics language this construction corresponds to the topological condensate [55] of the tensor product defect $T_{Q}\{1\}$ and $\tilde{I}^{0}$, triggered by the fermionic open-string cohomology element corresponding to $\tilde{r}$. Note that here $T_{Q}\{1\}$ denotes the anti-defect of $T_{Q}$ whose $R$-charges have been shifted by 1 . By this spectral flow, the bosonic morphism $\tilde{r}$ has become fermionic [56]. The final result of this condensation process yields the matrix factorization $C_{Q}$ for the conifold defect, which more concretely reads

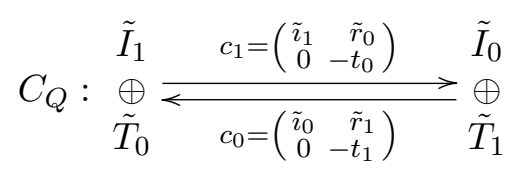

Note that due to the constant entries in the topological tachyon $\tilde{r}$, this matrix factorization can always be reduced to smaller rank by removing trivial brane-anti-brane pairs [57].

In this way we obtain candidates for the Landau-Ginzburg defect realizations of general conifold monodromies. In the next section we will show that they indeed give rise to the desired Fourier-Mukai transformations $\Phi^{\mathcal{K}_{\mathcal{Q}}^{\mathrm{C}}}$ with kernels (5.1) when transported into the large volume phase. 


\subsection{Transport to the large volume phase}

In order to transport the conifold defect (5.6) to the large volume phase we again follow the steps used in Section 4.4. We first observe that if two matrix factorizations $P$ of $W\left(x_{i}\right)$ and $Q$ of $W\left(y_{i}\right)$ give rise to large volume complexes $\mathcal{P}$ and $\mathcal{Q}$ when transported into the large volume phase through phase boundaries $N$ and $N^{\prime}$, respectively, the tensor product $P \otimes Q$ gives rise to the exterior product $\mathcal{P} \otimes \mathcal{Q}$ when transported to large volume through the phase boundary segment $N \times N^{\prime}$. This can directly be seen by comparing the unbounded complex obtained from the lift of the tensor product $P \otimes Q$ to the exterior product of the two unbounded complexes $\mathcal{P}$ and $\mathcal{Q}$. This general relation can be formulated as

$$
\operatorname{LV}_{N}(P) \otimes \operatorname{LV}_{N^{\prime}}(Q) \cong \operatorname{LV}_{N \times N^{\prime}}(P \otimes Q) .
$$

Furthermore, we use the relation (A.1)

$$
\operatorname{LV}_{-N}\left(P^{*}\right) \cong\left(\operatorname{LV}_{N}(P)\right)^{\vee} \otimes \mathcal{O}_{X}(d)\{-1\}
$$

between duality of matrix factorizations at the Landau-Ginzburg point and duality of complexes of coherent sheaves at large volume derived in Appendix A. Here $-N$ is the phase boundary component whose associated charge window is the dual $-\mathcal{N}$ of the one $\mathcal{N}$ associated to $N$.

Using these two relations, one easily sees that if the matrix factorization $Q$ corresponds to the large volume complex $\mathcal{Q}$ when transported from the Landau-Ginzburg to the large volume phase through the phase boundary component $N, T_{Q}$ gives rise to

$$
\mathrm{LV}_{N \times N^{*}} \cong \mathcal{Q} \otimes\left(\mathcal{Q}^{\vee} \otimes \mathcal{O}_{X}(-d)\{1\}\right)
$$

upon parallel transport through the phase boundary segment $N \times N^{*}$.

Using that the identity defect transports to the large volume complex

$$
L V_{N \times N^{*}}\left(\tilde{I}^{0}\right) \cong \mathcal{O}_{\Delta X}(-d)\{1\}
$$

which was shown in Section 4.3, we finally arrive at the large volume complex

$$
\operatorname{LV}_{N \times N^{*}}\left(C_{Q}\right) \cong \operatorname{Cone}\left(r: \mathcal{Q} \otimes\left(\mathcal{Q}^{\vee} \otimes \mathcal{O}_{X}(-d)\{1\}\right) \rightarrow \mathcal{O}_{\Delta X}(-d)\{1\}\right)
$$


arising by transporting the defect $C_{Q}$ of (5.6) through the phase boundary segment $N \times N^{*}$ to the large volume phase. Here, the restriction map $r$ arises in the following way: In the Landau-Ginzburg phase the corresponding morphism $\tilde{r}$ of matrix factorizations is induced from the identity map in $\operatorname{Hom}(Q, Q)$. As a result the large volume counterpart must be induced from the identity morphism $\operatorname{Hom}(\mathcal{Q}, \mathcal{Q})$ as well. This, however, is precisely the definition of the restriction map $r$ in Equation (5.1).

Tensoring the large volume kernel (5.12) by $\mathcal{O}_{X} \otimes \mathcal{O}_{X}(d)\{-1\}$ to obtain the associated Fourier-Mukai kernel (cf. (3.38)), we indeed find that the defect $C_{Q}$ at the Landau-Ginzburg point realizes the Fourier-Mukai transformation $\Phi^{\mathcal{K}_{\mathcal{Q}}^{\mathrm{C}}}$ on the category of the large volume B-branes which is associated to the conifold kernel $\mathcal{K}_{\mathcal{Q}}^{\mathrm{C}}$. This shows that $C_{Q}$ indeed represents the monodromy around the conifold point at which the D-brane associated to $Q$ becomes massless.

\section{Discussion}

In this paper we have discussed B-brane monodromy transformations from the world-sheet point of view. Our construction of monodromies can be understood as an application of the idea put forward in [12] that the effect which bulk perturbations of conformal field theories have on boundary conditions imposed on the world-sheet boundaries can be realized by fusion with specific defects associated to the perturbations.

We have constructed the defects corresponding to deformations along closed loops in Kähler moduli space of non-linear $\sigma$-models, which start and end at Landau-Ginzburg points. At these points the study of B-type defects and D-branes is particularly simple, because they can be elegantly described by means of matrix factorizations.

Using the description of B-type $\mathrm{D}$-branes in gauged linear $\sigma$-models developed in [22] we have shown that the action of any B-type defect at the Landau-Ginzburg point is realized by a Fourier-Mukai transformation on the B-brane category at large volume. Moreover, the particular FourierMukai transformations arising in this way from our monodromy defects indeed agree with the ones obtained from a more target space geometric approach to monodromies in $[13,14,16]$.

This gives further support to the idea that the effect of bulk perturbations on boundary conditions can be efficiently described using defects. A different 
type of examples, namely relevant flows between $N=2$ minimal models has been analysed in [12].

We have focused our considerations on non-linear $\sigma$-models on CalabiYau hypersurfaces in projective space, and also on the part of their Kähler moduli spaces which can be realized by means of a gauged linear $\sigma$-model with U(1) gauge group. It would be interesting to adapt our constructions to more general situations such as models with a more complicated phase structure. In particular, the lift to the gauged linear $\sigma$-model allows to derive all defects arising from monodromies around singularities in Kähler moduli space which are captured by the GLSM, by transporting the identity defect around the respective loops in Kähler moduli space. In this way, also more complicated monodromy groups could be analysed.

Our finding that fusion of defects at Landau-Ginzburg points is realized by means of Fourier-Mukai transformations in the large volume regime can also be turned around to provide a complementary perspective on functors on B-brane categories. Fusion of defects in Landau-Ginzburg models seems to be conceptually simpler and in some cases easier to work with in practice than the corresponding Fourier-Mukai transformations at large volume. As the example of the "quantum symmetry defects" discussed in Section 4.1 strikingly demonstrates, there are functors which seem to be rather complicated at large volume but have a very simple defect realization at the Landau-Ginzburg point.

In particular, the Landau-Ginzburg realization provides a new and potentially simpler method to calculate D-brane monodromies [14, 15, 31]. Interesting cases to study include the action of monodromies on D0-branes supported at specific points of Calabi-Yau target spaces, as well as examples exhibiting K-theory torsion.

\section{Acknowledgments}

H.J. and D.R. would like to thank the Banff International Research Station and the organizers of the workshop on matrix factorizations for a stimulating conference. I.B. thank the NHETC at Rutgers University for hospitality. We would also like to thank E. Diaconescu, M. Herbst and K. Hori for useful discussions. The work of I.B. is supported by a EURYI award of the European science foundation and furthermore by the Marie-Curie network Forces Universe (MRTN-CT-2004-005104). H.J. is supported by the Stanford Institute for Theoretical Physics. D.R. is supported by a DFG research fellowship and partially by DOE-grant DE-FG02-96ER40959. 


\section{Appendices}

\section{A Dual matrix factorizations and dual complexes}

Lifting a matrix factorization $P$ and its dual $P^{*}$ to the GLSM and transporting it into the large volume phase through the phase boundary component $N$ with associated charge window $\mathcal{N}$ and its dual $N^{*}$ with charge window $-\mathcal{N}$, respectively, one obtains two complexes $\mathcal{P}:=\operatorname{LV}_{N}(P)$ and line $\mathcal{P}:=\operatorname{LV}_{N^{*}}\left(P^{*}\right)$. In this Appendix we will show that they satisfy the following relations:

$$
\begin{aligned}
\operatorname{LV}_{N}(P) & \cong\left(\operatorname{LV}_{N^{*}}\left(P^{*}\right)\right)^{\vee} \otimes \mathcal{O}_{X}(-d)\{1\} \\
\left(\operatorname{LV}_{N}(P)\right)^{\vee} & \cong \operatorname{LV}_{N^{*}}\left(P^{*}\right)^{\vee} \otimes \mathcal{O}_{X}(d)\{-1\} .
\end{aligned}
$$

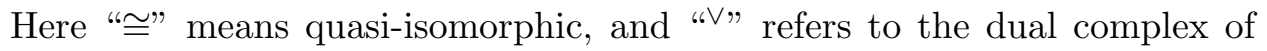
coherent sheaves.

The dual $P^{*}$ of the matrix factorization $P$ is defined in Equation (3.32), and it carries the duals $\rho^{*},\left(\rho^{R}\right)^{*}$ of the representations $\rho$ and $\rho^{R}$ of the orbifold group $\Gamma=\mathbb{Z}_{d}$ and the $R$-symmetry group $\mathrm{U}(1)_{R}$ defined on $P$ :

$$
\left(\rho^{*}\right)_{s}(\gamma)=\left(\rho_{s}\right)^{*}\left(\gamma^{-1}\right), \quad\left(\left(\rho^{R}\right)^{*}\right)_{s}(\varphi)=\left(\rho^{R}\right)_{s}^{*}(-\varphi) .
$$

As reviewed in Section 3 the large radius complex, $\mathcal{P}=\operatorname{LV}_{N}(P)$ obtained by transporting the Landau-Ginzburg B-brane represented by the matrix factorization $P$ through the phase boundary component $N$ into the large volume phase has the general form

$$
\mathcal{P}: \cdots \longrightarrow \mathcal{P}^{k-1} \stackrel{\wp_{k-1}}{\longrightarrow} \mathcal{P}^{k} \stackrel{\wp_{k}}{\longrightarrow} \mathcal{P}^{k+1} \stackrel{\wp_{k+1}}{\longrightarrow} \cdots .
$$

The sheaves $\mathcal{P}^{k}$ which appear in the complex at grading $k$ are given by

$$
\mathcal{P}^{k}=\bigoplus_{i \in A_{k}} \mathcal{O}_{X}\left(\frac{d}{2}\left(k-r_{i}\right)+q_{i}\right),
$$

in terms of the GLSM $R$-charges $r_{i}$ defined in Section 3.2 and the index sets

$$
A_{k}=\left\{i \mid 0 \leq \frac{1}{2}\left(k-r_{i}\right) \in \mathbb{Z}\right\} .
$$

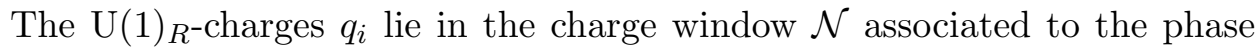
boundary component $N$. Finally, the maps $\wp_{k}$ are truncations of the maps $p_{s}$ defining the matrix factorizations $P$. 
The index set $A_{k}$ arises from the fact that in order to go to the large volume phase we need to integrate out the field $\mathfrak{p}$. On the boundary however, the field $\mathfrak{p}$ gives rise to a boundary interaction, which generates a Fock space for each index $i[22]$. The Fock space vacua have $U(1) \times U(1)_{R}$ charges $\left(q_{i}, r_{i}\right)$, whereas their $j$ th excited states have $U(1) \times U(1)_{R}$ charges $\left(q_{i}+\right.$ $\left.j d, r_{i}+2 j\right)$. The index set $A_{k}$ is a bookkeeping device for all the states in the Fock spaces. At large volume these states give rise to the line bundles $\mathcal{O}_{X}\left(q_{i}+j d\right)$ in the complex representing the D-brane under consideration.

There are a few comments in order. First, we observe that the complex (A.3) is bounded to the left because for sufficiently small $k$ the index sets $A_{k}$ are all empty. On the other hand, for $k$ sufficiently large the complex becomes two-periodic in the sense that $\wp_{k}=p_{k \bmod 2}$.

Analogously, lifting dual matrix factorization $P^{*}$ to the GLSM and transporting it through the phase boundary component $N^{*}$ corresponding to the dual charge window $\mathcal{N}^{*}=-\mathcal{N}$, the resulting complex reads

$$
\overline{\mathcal{P}}: \cdots \longrightarrow \overline{\mathcal{P}}^{k-1} \stackrel{\bar{\wp}_{k-1}}{\longrightarrow} \overline{\mathcal{P}}^{k} \stackrel{\bar{\wp}_{k}}{\longrightarrow} \overline{\mathcal{P}}^{k+1} \stackrel{\bar{\wp}_{k+1}}{\longrightarrow} \cdots,
$$

with

$$
\overline{\mathcal{P}}^{k}=\bigoplus_{i \in A_{k}^{*}} \mathcal{O}_{X}\left(\frac{d}{2}\left(k+r_{i}\right)-q_{i}\right), \quad A_{k}^{*}=\left\{i \mid 0 \leq \frac{1}{2}\left(k+r_{i}\right) \in \mathbb{Z}\right\} .
$$

Here the signs of the $\mathrm{U}(1)$ - and $\mathrm{U}(1)_{R}$-charges are reversed relative to the ones appearing in the definition of $\mathcal{P}$, because $P^{*}$ carries the dual representations of $\Gamma=\mathbb{Z}_{d}$ and $\mathrm{U}(1)_{R}$, and $P^{*}$ was lifted in a way compatible with the dual charge window $-\mathcal{N}$. The maps $\bar{\wp}_{k}$ in this complex are now truncations of the maps $\left(p^{*}\right)_{0}$ and $-\left(p^{*}\right)_{1}$ defining the dual $P^{*}$ of the matrix factorization $P$.

In order to determine the relation between the complexes (A.6) and (A.3) we form the dual complex, $\overline{\mathcal{P}}^{\vee}$ :

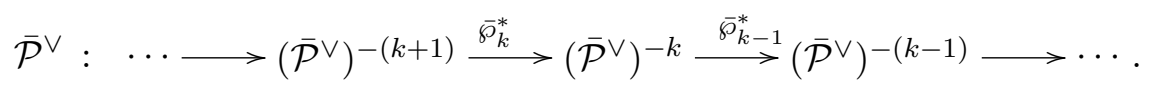

The maps $\bar{\wp}_{k}^{*}$ in the dual complex are induced by the maps $\left(p_{s}^{*}\right)^{*}=p_{s}$. The coherent sheaves in the dual complex at grading $-k$ are the dual sheaves of the original complex at grading $k$. Therefore, for the coherent sheaves in 
the dual complex at grading $k$ we arrive at

$$
\left(\overline{\mathcal{P}}^{\vee}\right)^{k}=\bigoplus_{i \in A_{k}^{* \vee}} \mathcal{O}_{X}\left(\frac{d}{2}\left(k-r_{i}\right)+q_{i}\right), \quad A_{k}^{* \vee}=\left\{i \mid 0 \geq \frac{1}{2}\left(k-r_{i}\right) \in \mathbb{Z}\right\} .
$$

By construction this dual complex is bounded to the right and two-periodic to the left with $\left(\varsigma^{*}\right)_{k}=p_{k \bmod 2}$ for $k$ sufficiently small. Note that the left two-periodic part of $\bar{P}^{\vee}$ is given by the maps $p_{s}$, as is the right two-periodic part of $\mathcal{P}$. Thus, it is tempting to combine these two complexes to form a single two-periodic complex unbounded to both sides. This is not quite possible however, because the index sets $A_{k}$ and $A_{k}^{* \vee}$ are not complementary. But this can be easily remedied by tensoring the complex $\overline{\mathcal{P}}^{\vee}$ with the oneterm complex $\mathcal{O}_{X}(-d)\{2\}$. The resulting complex

$$
\mathcal{F}:=\overline{\mathcal{P}}^{\vee} \otimes \mathcal{O}_{X}(-d)\{2\}: \cdots \longrightarrow \mathcal{F}^{k-1} \longrightarrow \mathcal{F}^{k} \longrightarrow \mathcal{F}^{k+1} \longrightarrow \cdots,
$$

now consists of sheaves

$$
\mathcal{F}^{k}=\bigoplus_{i \in B_{k}} \mathcal{O}_{X}\left(\frac{d}{2}\left(k-r_{i}\right)+q_{i}\right), \quad B_{k}=\left\{i \mid 0>\frac{1}{2}\left(k-r_{i}\right) \in \mathbb{Z}\right\}
$$

defined by index sets $B_{k}$ which are complementary to $A_{k}$.

Let us now analyse the consequences of this observation. As indicated we can now combine the two complexes $\mathcal{P}$ and $\overline{\mathcal{P}}^{\vee} \otimes \mathcal{O}_{X}(-d)\{2\}$ to form the announced unbounded two-periodic complex, which we denote by $\mathcal{Z}$. This is achieved in two steps. We first take the direct sum of these two complexes and then supplement the maps, which we collectively denote by $\delta \wp$ in the resulting complex at various gradings in order to obtain the desired two-periodicity. The described procedure, however, is simply the cone construction, i.e., the resulting complex $\mathcal{Z}$ is formally given by

$$
\mathcal{Z}=\text { Cone }\left(\delta_{\wp}: \overline{\mathcal{P}}^{\vee} \otimes \mathcal{O}_{X}(-d)\{1\} \rightarrow \mathcal{P}\right)
$$

But this complex is nothing but the unbounded two-periodic complex defined by the matrix factorization $P$, with maps $z_{k}=p_{k \bmod 2}$. Therefore, for all $k$ the image of the map $z_{k}$ equals the kernel of the map $z_{k+1}$, and hence the complex $\mathcal{Z}$ has no cohomology and is quasi-isomorphic to the null complex. This in turn implies that the constituents $\overline{\mathcal{P}}^{\vee} \otimes \mathcal{O}_{X}(-d)\{1\}$ and $\mathcal{P}$ are quasi-isomorphic to each other, which implies the advocated relations (A.1). 


\section{B Restriction map tachyon}

Here we derive the bosonic morphism $\tilde{r}$ between the tensor product matrix factorizations $T_{\tilde{Q}}$ defined in (5.4) and the matrix factorization $\tilde{I}^{0}$ representing the identity defect. It is an element of the BRST-cohomology group $\mathcal{H}_{\text {orb }}^{0}\left(T_{\tilde{Q}}, \tilde{I}^{0}\right)$ which is induced from the identity map in $\mathcal{H}_{\text {orb }}^{0}(\tilde{Q}, \tilde{Q})$ by means of the folding isomorphism (3.31). First, we construct the cohomology element $r=\left(r_{0}, r_{1}\right)$ of the corresponding matrix factorizations in the unorbifolded theory. It arises in the following commutative diagram:

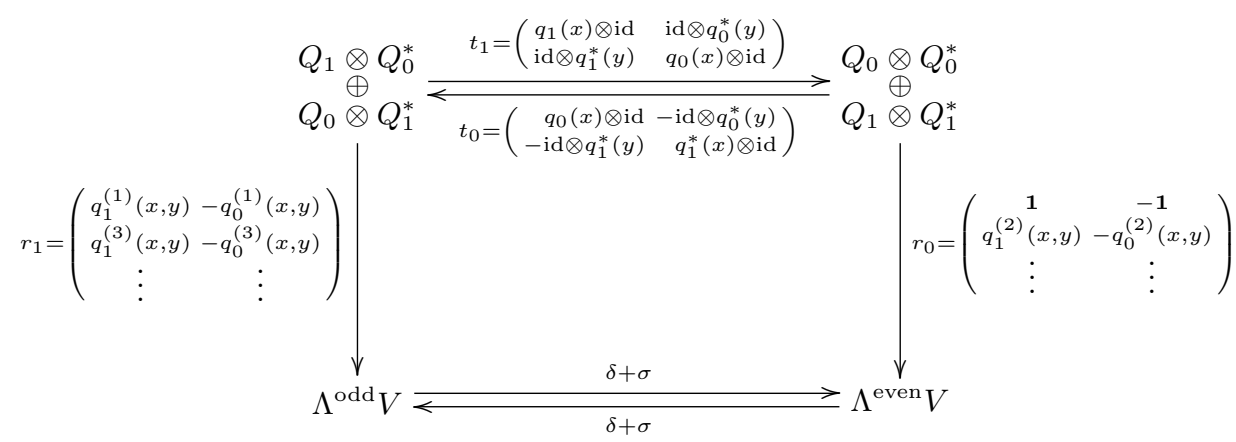

In order to construct all the entries of the matrices $\left(r_{0}, r_{1}\right)$ we first observe that the map $\delta$ acts on the module $\Lambda^{*} V$ as a differential obeying $\delta^{2}=0$. Thus we can analyse its cohomology. The only non-trivial cohomology is in degree 0 , and it is given by $\mathbb{C}\left[x_{i}, y_{i}\right] /\left(x_{1}-y_{1}, \ldots, x_{N}-y_{N}\right)$.

This allows us now to recursively define the entries of the matrices $r_{0}$ and $r_{1}$. The first entries, $q_{0}^{(1)}$ and $q_{1}^{(1)}$, of the matrix $r_{1}$ are defined by

$$
\delta\left(q_{s}^{(1)}(x, y)\right)=q_{s}(x)-q_{s}(y), \quad s=0,1
$$

Note that the right-hand sides vanish for $x=y$ and are therefore $\delta$-exact. Hence these two equations define $q_{s}^{(1)}$ up to unimportant $\delta$-closed one-forms.

Then the entries $q_{s}^{(k)}$ for $k \geq 2$ are given recursively by

$$
\begin{aligned}
\delta\left(q_{s}^{(k)}(x, y)\right)= & -\sum_{i} A_{i}(x, y) e_{i} \wedge q_{s}^{(k-2)}(x, y) \\
& +q_{s}^{(2 k-1)}(x, y) q_{s}(x)-q_{s}(y) q_{s+1}^{(2 k-1)}(x, y),
\end{aligned}
$$


with $q_{s}^{(0)}=(-1)^{s+1} \mathbf{1}$. Similarly to the case $k=1$, it is straightforward to check that the right-hand sides of all the equations (B.3) are $\delta$-closed. Moreover, due to the fact that there is no non-trivial cohomology at higher degree, $\delta$-closedness implies $\delta$-exactness. Thus the relations (B.3) also can be solved recursively to define (again up to unimportant closed contributions) all the other entries of $r_{0}$ and $r_{1}$.

With the definitions of the entries $r_{0}$ and $r_{1}$ at hand it is straightforward to check that the diagram (B.1) is indeed commutative. In addition, the constant entries in the matrix $r_{0}$ ensure that $\left(r_{1}, r_{0}\right)$ defines a non-trivial morphism in $\mathcal{H}^{0}\left(Q \otimes Q^{*}, I\right)$. In fact, it can be identified with the identity morphism in $\mathcal{H}^{0}(Q, Q)$.

Eventually we need the corresponding morphism $\tilde{r} \in \mathcal{H}_{\text {orb }}^{0}\left(\tilde{Q}^{\rho} \otimes \tilde{Q}^{\rho *}, \tilde{I}^{0}\right)$ in the orbifold category. The orbifold construction for matrix factorization has been reviewed in Section 4.1. The upshot is that we obtain the morphism $\tilde{r}$ for the equivariant matrix factorization $\tilde{Q}$ in the basis which diagonalizes the action of the orbifold group $\Gamma \times \Gamma$ simply by replacing the variables $x$ and $y$ in $r$ by the matrices $x$ id and $y \tau$ introduced in Section 4.1, respectively, and then projecting on its $\Gamma \times \Gamma$ invariant part:

$$
\tilde{r}(x, y)=(r(x \mathrm{id}, y \tau))^{\Gamma \times \Gamma}
$$

\section{References}

[1] C. Bachas and I. Brunner, Fusion of conformal interfaces, JHEP 02 (2008), 085; arXiv:0712.0076 [hep-th].

[2] V. B. Petkova and J. B. Zuber, Generalised twisted partition functions, Phys. Lett. B504 (2001), 157-164; hep-th/0011021.

[3] C. Bachas, J. de Boer, R. Dijkgraaf and H. Ooguri, Permeable conformal walls and holography, JHEP 06 (2002), 027; hep-th/0111210.

[4] J. Fröhlich, J. Fuchs, I. Runkel and C. Schweigert, Kramers-Wannier duality from conformal defects, Phys. Rev. Lett. 93 (2004), 070601; cond-mat/0404051.

[5] C. Bachas and M. Gaberdiel, Loop operators and the Kondo problem, JHEP 11 (2004), 065; hep-th/0411067.

[6] J. Fröhlich, J. Fuchs, I. Runkel and C. Schweigert, Duality and defects in rational conformal field theory, Nucl. Phys. B763 (2007), 354-430; hep-th/0607247.

[7] T. Quella, I. Runkel and G. M. T. Watts, Reflection and transmission for conformal defects, JHEP 04 (2007), 095; hep-th/0611296. 
[8] A. Alekseev and S. Monnier, Quantization of Wilson loops in WessZumino-Witten models, JHEP 08 (2007), 039; hep-th/0702174.

[9] J. Fuchs, M. R. Gaberdiel, I. Runkel and C. Schweigert, Topological defects for the free boson CFT, arXiv:0705.3129 [hep-th].

[10] I. Runkel, Perturbed defects and T-systems in conformal field theory, arXiv:0711.0102 [hep-th].

[11] I. Brunner and D. Roggenkamp, B-type defects in Landau-Ginzburg models, JHEP 08 (2007), 093; arXiv:0707.0922 [hep-th].

[12] I. Brunner and D. Roggenkamp, Defects and bulk perturbations of boundary Landau-Ginzburg orbifolds, arXiv:0712.0188 [hep-th].

[13] R. P. Horja, Hypergeometric functions and mirror symmetry in toric varieties, math/9912109.

[14] P. S. Aspinwall and M. R. Douglas, D-brane stability and monodromy, JHEP 05 (2002), 031; hep-th/0110071.

[15] J. Distler, H. Jockers and H.-J. Park, D-brane monodromies, derived categories and boundary linear sigma models, hep-th/0206242.

[16] P. S. Aspinwall, R. L. Karp and R. P. Horja, Massless D-branes on Calabi-Yau threefolds and monodromy, Commun. Math. Phys. 259 (2005), 45-69; hep-th/0209161.

[17] M. Kontsevich, Lecture at Rutgers University, unpublished (1996).

[18] A. Kapustin and Y. Li, D-branes in Landau-Ginzburg models and algebraic geometry, JHEP 12 (2003), 005; hep-th/0210296.

[19] I. Brunner, M. Herbst, W. Lerche and B. Scheuner, Landau-Ginzburg realization of open string TFT, JHEP 11 (2006), 043; hep-th/0305133.

[20] M. Khovanov and L. Rozansky, Topological Landau-Ginzburg models on a world-sheet foam, hep-th/0404189.

[21] D. Orlov, Triangulated categories of singularities and D-branes in Landau-Ginzburg models, math/0302304.

[22] M. Herbst, K. Hori and D. Page, Phases of $N=2$ theories in $1+1$ dimensions with boundary, arXiv:0803.2045 [hep-th].

[23] P. S. Aspinwall, The Landau-Ginzburg to Calabi-Yau dictionary for D-branes, hep-th/0610209.

[24] M. Oshikawa and I. Affleck, Boundary conformal field theory approach to the critical two-dimensional Ising model with a defect line, Nucl. Phys. B495 (1997), 533-582; cond-mat/9612187.

[25] E. Witten, Phases of $N=2$ theories in two dimensions, Nucl. Phys. B403 (1993), 159-222; hep-th/9301042.

[26] M. R. Douglas, D-branes, categories and $N=1$ supersymmetry, J. Math. Phys. 42 (2001), 2818-2843; hep-th/0011017. 
[27] C. I. Lazaroiu, Generalized complexes and string field theory, JHEP 06 (2001), 052; hep-th/0102122.

[28] P. S. Aspinwall and A. E. Lawrence, Derived categories and zero-brane stability, JHEP 08 (2001), 004; hep-th/0104147.

[29] A. Strominger, Massless black holes and conifolds in string theory, Nucl. Phys. B451 (1995), 96-108; hep-th/9504090.

[30] P. S. Aspinwall, D-branes on Calabi-Yau manifolds, hep-th/0403166.

[31] H. Jockers, D-brane monodromies from a matrix-factorization perspective, JHEP 02 (2007), 006; hep-th/0612095.

[32] D.-E. Diaconescu, Enhanced D-brane categories from string field theory, JHEP 06 (2001), 016; hep-th/0104200.

[33] P. Mayr, Phases of supersymmetric D-branes on Kaehler manifolds and the McKay correspondence, JHEP 01 (2001), 018; hep-th/0010223.

[34] K. Hori, Linear models of supersymmetric D-branes, Symplectic geometry and mirror symmetry, (Seoul 2000); hep-th/0012179.

[35] S. Fredenhagen, M. R. Gaberdiel and C. A. Keller, Bulk induced boundary perturbations, J. Phys. A40 (2007), F17; hep-th/0609034.

[36] S. K. Ashok, E. Dell'Aquila and D.-E. Diaconescu, Fractional branes in Landau-Ginzburg orbifolds, Adv. Theor. Math. Phys. 8 (2004), 461$513 ;$ hep-th/0401135.

[37] K. Hori and J. Walcher, F-term equations near Gepner points, JHEP 01 (2005), 008; hep-th/0404196.

[38] S. Govindarajan and T. Jayaraman, D-branes, exceptional sheaves and quivers on Calabi-Yau manifolds: from Mukai to McKay, Nucl. Phys. B600 (2001), 457-486; hep-th/0010196.

[39] A. Tomasiello, D-branes on Calabi-Yau manifolds and helices, JHEP 02 (2001), 008; hep-th/0010217.

[40] H. Enger, A. Recknagel and D. Roggenkamp, Permutation branes and linear matrix factorisations, JHEP 01 (2006), 087; hep-th/0508053.

[41] S. Govindarajan and T. Jayaraman, Boundary fermions, coherent sheaves and D-branes on Calabi-Yau manifolds, Nucl. Phys. B618 (2001), 50-80; hep-th/0104126.

[42] S. Hellerman, S. Kachru, A. E. Lawrence and J. McGreevy, Linear sigma models for open strings, JHEP 07 (2002), 002; hep-th/0109069.

[43] S. Govindarajan and H. Jockers, Effective superpotentials for B-branes in Landau-Ginzburg models, JHEP 10 (2006), 060; hep-th/0608027.

[44] D. Eisenbud, The geometry of syzygies: a second course in commutative algebra and algebraic geometry, Graduate Texts in Mathematics, 229, Springer-Verlag, Berlin, 2005. 
[45] D. Eisenbud, Commutative algebra with a view toward algebraic geometry, Graduate Texts in Mathematics, 150, Springer-Verlag, Berlin, 1995.

[46] M. R. Douglas, B. Fiol and C. Römelsberger, The spectrum of BPS branes on a noncompact Calabi-Yau, JHEP 09 (2005), 057; hep-th/0003263.

[47] D. Orlov, Equivalences of derived categories and K3 surfaces, J. Math. Sci. 84(5) (1997), 1361-1381; alg-geom/9606006.

[48] T. Bridgeland, Equivalences of triangulated categories and FourierMukai transforms, Bull. London Math. Soc. 31(1) (1999), 25-34; math. AG/9809114.

[49] S. I. Gelfand and Y. I. Manin, Methods of homological algebra, Springer Monographs in Mathematics, Springer-Verlag, Berlin, 2002.

[50] B. R. Greene and Y. Kanter, Small volumes in compactified string theory, Nucl. Phys. B497 (1997), 127-145; hep-th/9612181.

[51] P. Candelas and X. C. de la Ossa, Comments on conifolds, Nucl. Phys. B342 (1990), 246-268.

[52] M. Kontsevich, Homological algebra of mirror symmetry, alg-geom/9411018.

[53] I. Brunner and J. Distler, Torsion D-branes in nongeometrical phases, Adv. Theor. Math. Phys. 5 (2002), 265-309; hep-th/0102018.

[54] P. Seidel and R. Thomas, Braid group actions on derived categories of coherent sheaves, Duke Math. 108 (2001), 37-108; math. AG/0001043.

[55] A. Sen, Tachyon condensation on the brane antibrane system, JHEP 08 (1998), 012; hep-th/9805170.

[56] E. Witten, D-branes and K-theory, JHEP 12 (1998), 019; hep-th/9810188.

[57] S. Govindarajan, H. Jockers, W. Lerche and N. P. Warner, Tachyon condensation on the elliptic curve, Nucl. Phys. B765 (2007), 240-286; hep-th/0512208. 
